\title{
Training spatial cognition enhances mathematical learning - a randomized study in 17.000 children
}

Nicholas Judd ${ }^{1}$, Torkel Klingberg ${ }^{1 *}$

${ }^{1}$ Department of Neuroscience, Karolinska Institutet, Stockholm, Sweden

*Corresponding author: Torkel Klingberg (torkel.klingberg@ki.se; https://orcid.org/0000-00023175-217) 


\section{Abstract}

Spatial and mathematical abilities are strongly associated. Here we analyzed data from 17,648 children, aged 6-8 years, who performed 7 weeks of mathematical training together with randomly assigned, spatial cognitive training with tasks demanding more of spatial manipulation (mental rotation or tangram), maintenance of spatial information (a visuo-spatial working memory task) or spatial, non-verbal reasoning. We found that what type of cognitive training children performed had a significant impact on mathematical learning, with training of visuo-spatial working memory and reasoning being the most effective. This large, community-based study shows that spatial cognitive training can result in transfer to academic abilities and that reasoning ability and maintenance of spatial information is relevant for math learning in young children. 


\section{Introduction}

Spatial ability is closely associated with performance in science, technology, engineering and mathematics ${ }^{1}$. For example, the ability to mentally rotate a figure in mind correlates with current performance in mathematics and predicts later learning in children ${ }^{1-3}$. Similar associations exist for other spatial tasks, including visuo-spatial working memory (vs-WM) ${ }^{4,5}$ and spatial, non-verbal reasoning (NVR) .

Consequently, it has been suggested that improving spatial abilities might be a way to enhance mathematical learning ${ }^{7-9}$, with some teacher organizations going as far as to place equal emphasis on spatial training as on numerosity ${ }^{10}$. However, the mechanisms of spatial training are still unclear, and there is a lack of large, randomized studies assessing its effect on mathematical performance ${ }^{9}$.

Spatial tasks involve at least three aspects: creating an internal spatial representation, maintaining it, and manipulating $\mathrm{it}^{11}$. A mental rotation task involves representation and manipulation, but minimal maintenance. A vs-WM task, on the other hand, puts a higher demand on maintenance, but less on manipulation.

This distinction is at the heart of two hypotheses on why training spatial abilities could transfer to improvements in mathematics. The first hypothesis is that manipulation is the critical aspect, and that rotation training therefore would be more effective than training on vs-WM $\mathrm{W}^{2,8}$. In the second hypothesis, maintenance of spatial information that is critical, and training on vs-WM should therefore be superior ${ }^{11,12}$. It's also possible, of course, that both hypotheses are wrong and spatial training does not transfer to mathematics at all.

Prior literature gives mixed support for both hypotheses of transfer. Several studies have shown a positive impact of rotation training on mathematics ${ }^{13-15}$ while others found no effect ${ }^{16-}$ ${ }^{18}$. Similarly, training of vs-WM improves mathematical outcomes in children in some studies ${ }^{19-}$ ${ }^{21}$, although there are also negative findings ${ }^{22,23}$. Given these mixed results, some suggest that cognitive training, in general, does not transfer at all to academic abilities ${ }^{24}$.

This broad lack of consensus in cognitive training literature could be due to inadequate statistical power ${ }^{25}$ along with meta-analyses combining different training methods, populations, and outcome measure ${ }^{26}$. Perhaps most importantly, there is a lack of large, randomized studies.

Here we report data from over 17,000 children who engaged in various forms of mathematical and cognitive training. We asked two questions that are of both theoretical and practical relevance: 1) Does spatial cognitive training impact mathematical learning, and if so, is it more effective to train mental rotation or vs-WM? 2) Are there inter-individual characteristics that predict the optimal type of cognitive training to enhance mathematics?

Although our main hypothesis is the contrast between mental rotation and vs-WM, we also included training of spatial, non-verbal reasoning (NVR). Non-verbal reasoning tasks rely heavily on one's ability to find visuo-spatial patterns and determine how these patterns spatially interrelate $^{27}$. They were also added based on the high correlation between non-verbal reasoning and mathematical ability ${ }^{25-27}$ and some evidence that such training can enhance problem-solving ability ${ }^{28-30}$.

We conducted our study by modifications on a freely available app (Vektor) using number line tasks to train mathematics. The app was used as an extra, voluntary activity in school, organized by teachers. When using the app, children spent half of their time with number line tasks, as the efficacy of this training was established for this app ${ }^{31}$ as well as for similar training using the number line $e^{32,33}$. The remaining time was allotted, by randomization, to the 
training of rotation tasks (2D mental rotation and tangram), vs-WM, or NVR (Fig. 1; SI Fig. 1 \& 2 ). In the $1 \mathrm{st}, 5$ th, and 7 th week, children performed self-administered tests of mathematics (addition, subtraction, and number comparison). For feasibility reasons, mathematical tests had to be self-administered, yet this allowed us to have one of the largest, most comprehensive (1220 hours of training) and ecologically valid training studies to date.

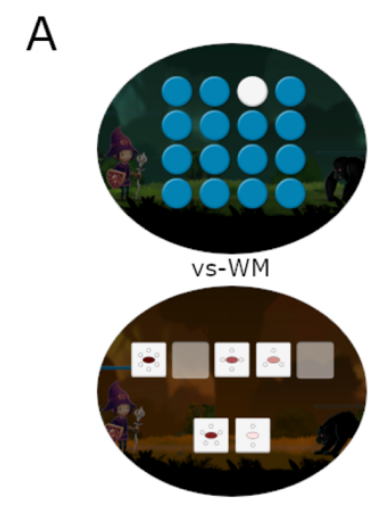

NVR
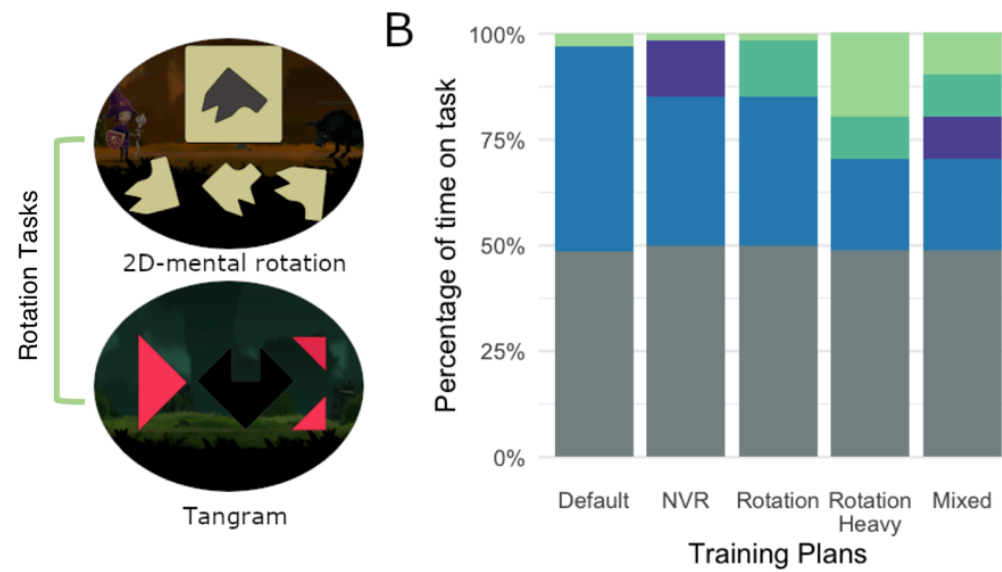

Training Tasks

Tangram 2D Rot NVR

vs-WM Numberline

Figure 1 A) Figures of training tasks; vs-WM: a visuo-spatial WM task where participants had to remember a sequence of dots on a $4 \times 4$ grid and after a delay respond to them in order; NVR: a spatial, non-verbal reasoning task in which subjects had to find spatial patterns among figures; 2D-mental rotation: a task in which children had to choose the shape that when rotated would fit into an empty silhouette; Tangram: a second rotation-task which children had to manipulate and rotate several pieces to fit into an empty silhouette $\boldsymbol{B}$ ) The percentage of time for each cognitive training task in each of the five training plans. The tasks 2D mental rotation (i.e., 2D Rot) and Tangram are classified as Rotation.

\section{Results}

Over the course of seven weeks, children completed cognitive training for either 20 or 33 minutes per day. Our final sample consisted of 17,648 children between the ages of six and eight, who on average completed 5,077 trials (standard deviation $(\mathrm{SD})=1,710$ ).

To measure baseline performance within each training domain, we designed the app to give every child an identical first week. Task performance for all tasks was moderately correlated with each other as well as with the mathematical transfer tests, consistent with the well-documented association between spatial cognition and mathematics (Fig. 2b). 


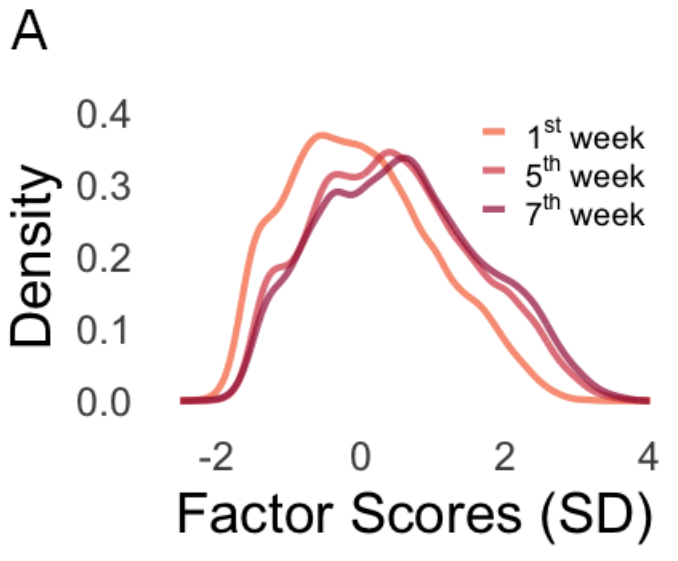

C

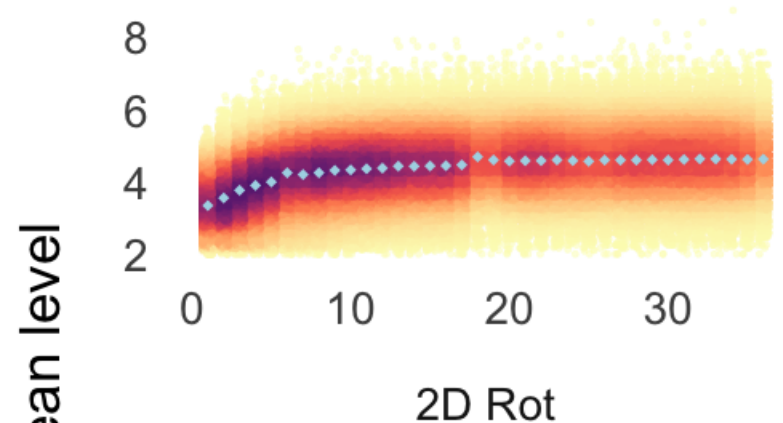

7.5

5.0

2.5

0.0

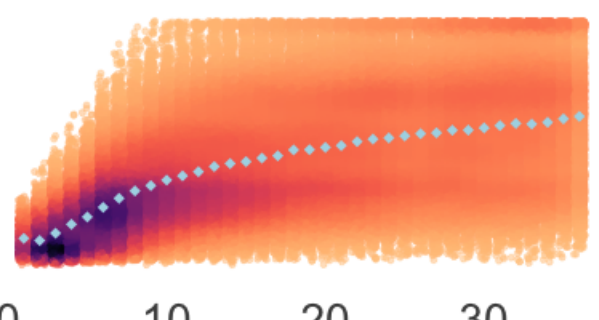

B

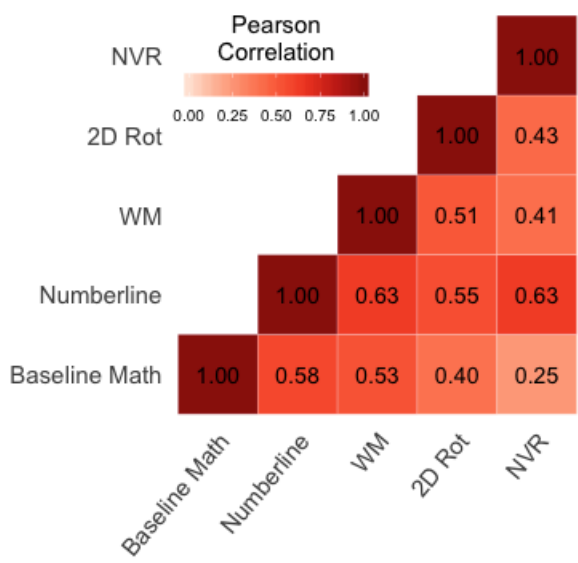

Numberline

100

50

0 0

10

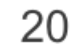

30

\section{NVR}

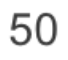

40

30

20

10

0

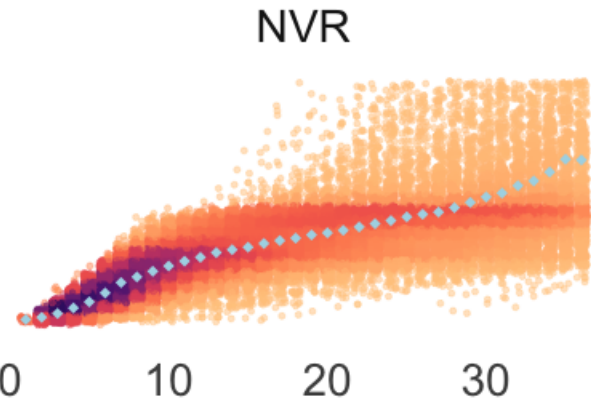

\section{Training Day}

Figure 2 A) Density plots of predicted factor scores from three tests of mathematics by test week (SI Fig. 4 shows individual tests). $\boldsymbol{B}$ ) Correlations of the mean correct level for the training tasks in the first week with baseline math (a subject-specific intercept of the math factor). All correlations are significant ( $\left.p^{\prime} s<.001\right)$. C) Training curves showing the density of children for each difficulty level and day of training. Light blue diamond's denote the mean correct level per day of each of the corresponding tasks.

Following this first week, individuals were randomly split into one of five training plans. Each plan had the same amount of mathematical training ( $\sim 50 \%$ of training-time) yet differed in proportions of cognitive training tasks (Fig. 1b; SI Table 1). All training tasks were adaptive, so children progressed to gradually more difficult levels (Fig. 2c; SI Fig. 3). There was a significant improvement, as judged by an increase in average difficulty level in all training tasks between 
the $1^{\text {st }}$ and $7^{\text {th }}$ week. The improvement (in SDs) was: vs-WM $=.55$, Number line $=0.77,2 \mathrm{D}$ mental rotation $=.81, \mathrm{NVR}=.77$, Tangram $=.58$.

We combined the performance on the three transfer tests using a confirmatory factor analysis (CFA), which fit the data well (CFI > .95, RMSEA <.08; SI Fig. 4 \& 5, SI Table 3). The average improvement from the 1 st to 5 th week was $0.45 \mathrm{SD}$, and to the 7 th week $0.56 \mathrm{SD}$ (Fig. 2a, SI Table 2), showing clear improvement but also suggesting a diminished return for the last two weeks of training compared to the prior weeks.

To estimate the validity of these on-line tests, we compared the performance of 106 children (age 6-8) who took both online and experimenter administered tests of addition, subtraction, and the WISC-IV verbal arithmetic's test, which also were combined using factor analysis. We found a high correlation $\left(\mathrm{r}=.70,95 \% \mathrm{CI}[.58-.78], \mathrm{p}<2.2 \times 10^{-16}\right)$ between factors from online and experimenter led tests, supporting the use of online tests as outcome measures (SI Fig. 6).

We proceeded to use the CFA scores in a linear, mixed-effects model with a random intercept and slope for each child (SI Table's 4A-D). The intercept and the slope were moderately correlated $(\mathrm{r}=.28)$, showing that children with higher starting scores improved more from training, i.e. a Matthew's effect.

To evaluate the differences between the spatial cognitive training tasks we used three complementary analyses: 1) an omnibus test of the effect of training plans (Fig. 1b), coded categorically; 2) a model accounting for the proportions of rotation training (including both mental rotation and tangram) and NVR within those plans, with the default plan (with $\sim 50 \%$ vsWM) as a reference (SI Table 1); and 3) modeling the size of the effect per minute spent with each of the cognitive tasks on the slope of mathematical improvement. All three analyses thus addressed the same question: if the type of cognitive training impacted mathematical learning, but analyzed cognitive training in terms of plans, categories or single tasks.

In the first analysis, a model with training plans interacting with test week predicted mathematical improvement significantly better than a model without $\left(\triangle \mathrm{AIC}=24, x^{2}(4)=32.24\right.$, $\left.\mathrm{p}=1.7 \times 10^{-6}\right)($ SI Table 4b-c; SI Fig. 7). The largest improvement was seen in the NVR group (0.602 units), and the smallest improvement in the Rotation heavy group (0.540 units), i.e. 11.5\% larger effect of training, or slightly more than the effect of increasing total training time (including number line) from 20 to 33 minutes per day (SI Table 4a).

In the second analysis, to evaluate the effect of differing amounts of cognitive training, we coded for time spent with rotation (combining time for 2D mental rotation and tangram training) or NVR (SI Table $4 \mathrm{~b} \& \mathrm{~d}$ ). In this model vs-WM was coded as the intercept, therefore the effects of NVR and rotation are in reference to active vs-WM training. Adding terms for NVR and rotation interacting with test week improved model fit $\left(\triangle \mathrm{AIC}=24 ; x^{2}(2)=28.23, \mathrm{p}=\right.$ $7.0 \times 10^{-7}$ ), showing that mathematical improvement depended on the amount of NVR and rotation training. NVR training showed a positive effect $(b=.023,95 \%$ CI $[.003-.042], p=$ $.024)$, in reference to only vs-WM training. In contrast, children with rotation training improved less $\left(b=-.023,95 \%\right.$ CI [-.033 - -.013], $\left.\mathrm{p}=6.6 \times 10^{-6}\right)$, again in comparison to those with vsWM instead. This shows that removing vs-WM training to incorporate rotation had a negative outcome on children mathematics, while NVR had a positive effect.

In order to evaluate if the two different rotation tasks (2D-rotation and tangram) had differential effects we performed a third analysis, where we evaluated the impact of each of the different cognitive tasks separately on mathematical outcomes. Subject-specific slopes of mathematical improvement were extracted. We then calculated the amount of time, in minutes, 
spent on different training tasks. These values were entered into a linear model, with the slope as the outcome and covarying for subjects' baseline math abilities (i.e., subject-specific intercepts).

The amount of time spent training NVR, vs-WM, 2D mental rotation and tangram all positively predicted mathematical improvement (all ps $<.05$ ). This test is in line with the previous two analyses, as amount of NVR training $(b=.030,95 \%$ CI $[.020-.039])$ showed the largest effect, followed by vs-WM $(b=.021,95 \% \mathrm{CI}[.017-.025])$ and then $2 \mathrm{D}$ mental rotation $(\mathrm{b}=.012,95 \%$ CI $[.004-.020])$ and Tangram $(\mathrm{b}=.008,95 \%$ CI $[.001-.016])$. The difference between 2D mental rotation and tangram was not significant (Wald Test; $\mathrm{F}(1)=0.34, \mathrm{p}=.56$ ), yet both differed when contrasted to vs-WM (p's <.05) and NVR (p's <.05).

All three analyses were thus consistent in finding a significant impact of the type of cognitive training performed, with significantly larger effects of vs-WM and NVR compared to rotation training for the math transfer tests (Fig. 3).

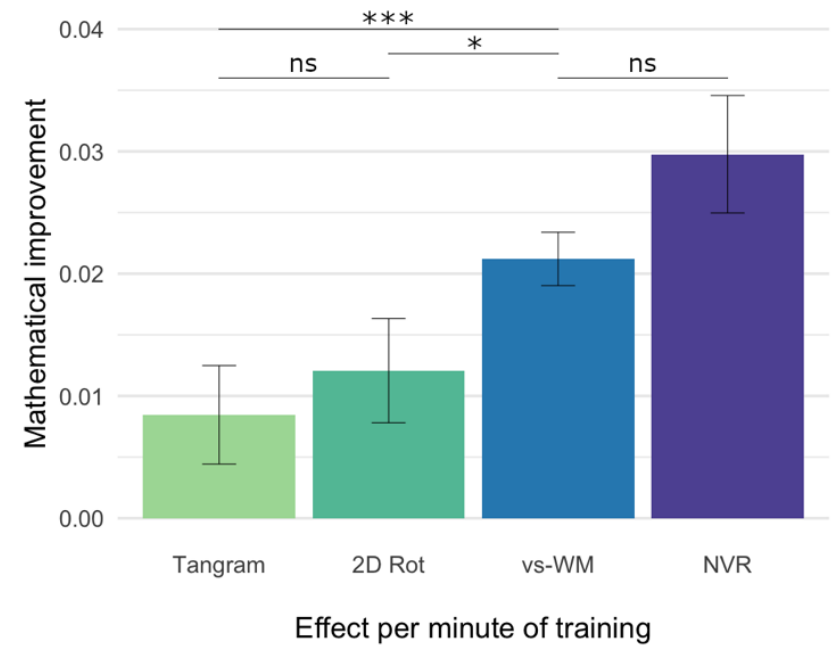

Figure 3 The impact of different types of training on interindividual mathematical improvement (i.e., random slopes) while correcting for subject's baseline mathematics (i.e., random intercepts). These coefficients are predicted from a mixed-effects model (see methods for further details).

Our second question was to assess if there were inter-individual characteristics at baseline that predicted the optimal type of cognitive training to enhance mathematics. For example, our previous analysis showed that rotation training is less effective on average, but it might be that rotation training is more effective for some children? Children were characterized based on cognitive and math performance in the first week (Fig. 2b). Both accuracy and average difficulty level were included, resulting in 8 predictor variables from the cognitive tasks (SI Table 5).

First, we evaluated predictors of mathematical improvement independent of the training plans. As shown by earlier analyses, baseline performance on the math tests (i.e. intercept) had a positive impact on improvement (i.e., Matthew's effect). However, after correcting for baseline mathematics, children with a lower than average level in number line improved more in mathematics over the course of training $\left(b=-.12,95 \%\right.$ CI $[-.15--.10]$, pFDR $\left.=9.3 \times 10^{-21}\right)$. Children with a higher level of NVR $\left(b=.03,95 \%\right.$ CI $\left.[.01-.05], \mathrm{pFDR}=1.7 \times 10^{-3}\right)$ and higher accuracy on vs-WM $\left(b=.05,95 \%\right.$ CI $\left.[.03-.06], \mathrm{pFDR}=9.7 \times 10^{-9}\right)$ also improved more.

Secondly, we tested if differing amounts of cognitive training (vs-WM, Rotation, or NVR) interacted with any of these baseline characteristics to predict mathematical improvement (Fig. 4). This analysis showed that children with initially higher vs-WM accuracy $(b=.006,95 \%$ 
CI $\left.[.002-.011], \mathrm{pFDR}=8.0 \times 10^{-3}\right)$ or lower NVR performance $(\mathrm{b}=-.007,95 \% \mathrm{CI}[-.012-$ $.002], \mathrm{pFDR}=2.0 \times 10^{-2}$ ), benefitted more from vs-WM training. Moreover, children with lower initial NVR levels benefitted more from NVR training $(b=-.014,95 \%$ CI [-.024 - -.004], pFDR $=2.2 \times 10^{-2}$ ). There were no significant interactions between any of the baseline performance measures and amount of time with rotation training.

A

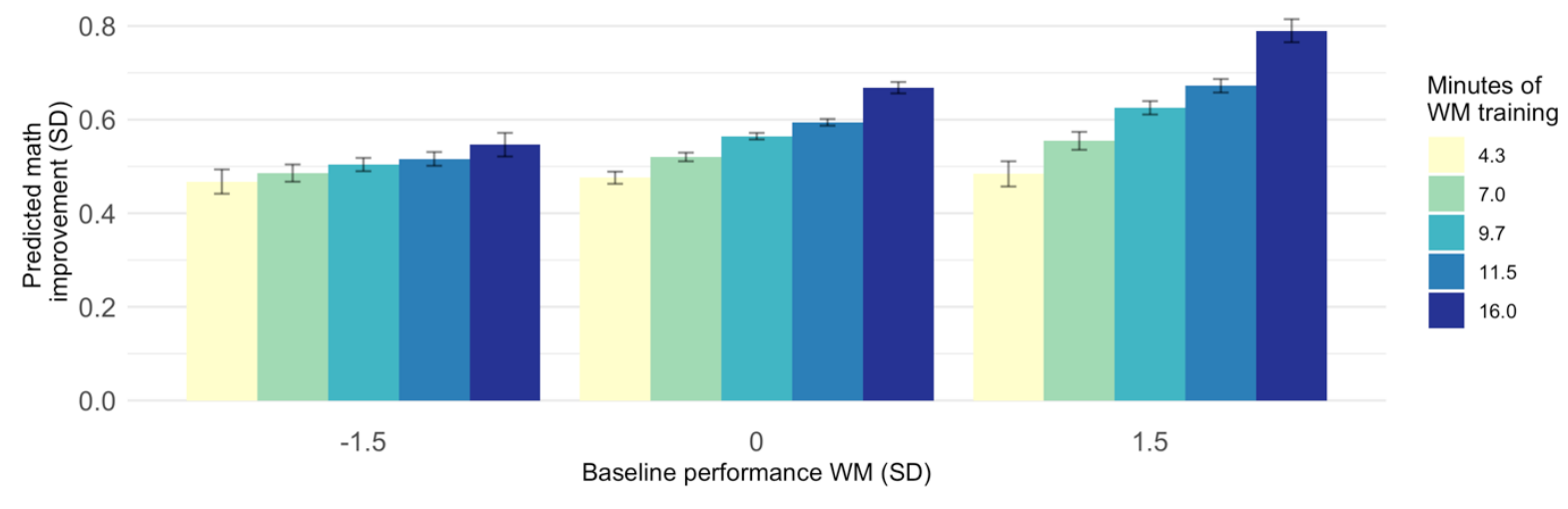

B

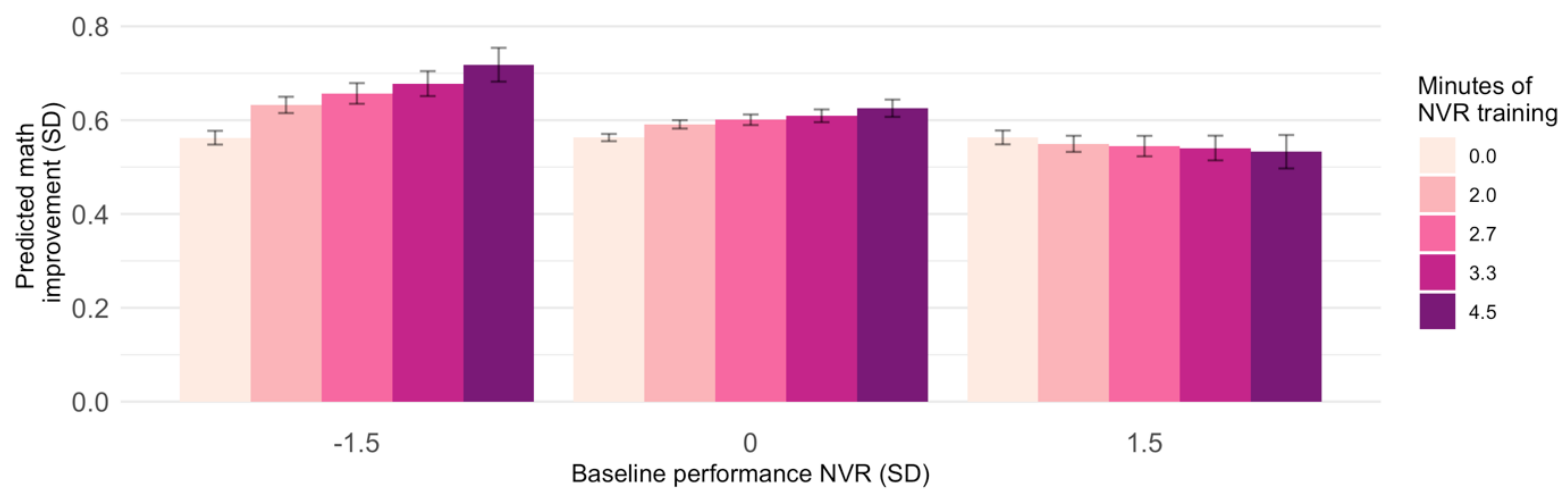

Figure 4 Predicted performance from the two significant models of interactions between baseline cognitive performance and spatial training: a) baseline WM performance interaction with amount of WM training; $b$ ) baseline NVR performance interaction with amount of NVR training. Baseline performance was measured from the first week of training, prior to randomization. The improvement in mathematics was estimated from the slopes of improvement over the three tests, extracted from the mixed effects model, and corrected for baseline mathematical performance.

\section{Discussion}

Here we report strong evidence that spatial cognitive training impacts mathematical learning in children. Taking the type of cognitive activity into account resulted in a model that was around twenty times more likely for predicting mathematical improvement than a model that did not account for them $(\triangle \mathrm{AIC}=24)$. 
Specifically, we found that training on vs-WM was more effective than both types of rotation training (2D mental rotation and tangram). This suggests that, when it comes to transfer to mathematics, the crucial aspect of spatial training is maintaining a spatial representation, rather than manipulating it. It is in line with the suggestions that a bottleneck for spatial cognition is the ability to maintain the spatial representation ${ }^{31-33}$, and that subject with problems in mental rotation "lose" the image they attempt to keep in mind.

A priori, we expected rotation training to have a positive impact on math learning. However, rotation turned out to have the smallest effect. Being the worst performing condition, rotation training can be considered an active and very strict control group for the other conditions. Our analysis showed that, compared to rotation training, both vs-WM and NVR training had a significant and meaningful impact on math learning. This is the largest randomized, controlled study to date showing that cognitive training results in transfer to academic abilities, adding evidence in favor of the view that cognitive abilities are malleable ${ }^{34-}$ ${ }^{36}$, and shows the relevance of such training for academic performance in an ecologically valid setting.

Our finding of transfer to mathematical learning is consistent with some prior studies ${ }^{19-21}$ but is at odds with some others, the largest negative study being that by Roberts and colleagues ${ }^{31}$. One reason for the discrepancies could be that the current study included a general population while the study by Roberts et al. included only children with low WM capacity (lowest 15 th percentile). As shown by the interaction analysis (Fig. 4a), and consistent with prior findings, the impact of vs-WM training depends heavily on baseline performance ${ }^{28-30}$. Such dependence on baseline characteristics needs to be taken into account in interpreting the existent literature as well as planning of future studies.

An interesting finding was the positive effect of NVR training. Although such training is much less researched, our finding is consistent with previous research showing NVR training to improve cognitive abilities and academic performance ${ }^{37}$. Our NVR task was similar to the Sequential Order task from the Leiter test Battery ${ }^{2,38}$. It would be of interest for future research to focus on specific mechanisms of transfer, study the relationship between NVR tasks and mathematic ${ }^{39,40}$, and to evaluate training on different types of fluid reasoning tasks.

Regarding inter-individual characteristics, children with higher initial vs-WM abilities, but lower NVR performance, benefited more from vs-WM training. The benefit of 16 vs 4.3 minutes of WM training differed 3-fold between the high- and low-WM groups (predicted gain of $.31 \mathrm{vs} .08 \mathrm{SD}$; Fig. $4 \mathrm{a}$ ). In contrast, there was a negative interaction between baseline NVR performance and amount of NVR training (Fig. 4b). However, it is important to note that the range of NVR training time was constrained to between 0 to 4.5 minutes. Whether the same pattern would be observed if NVR training time was expanded to $16 \mathrm{~min}$, remains to be seen. Critically, we did not find any inter-individual characteristics showing children to benefit from rotation training. These findings show how baseline characteristics can predispose children to benefit from specific types of training. 
Here we only randomized a minor part of training activities, as $70 \%$ of the time was identical for all children. Yet, slight changes in the cognitive content of $30 \%$ of the time, resulted in a 11.5\% difference in mathematical learning, with some tasks being 2-3 times more effective than others (Fig. 3). Because this study did not include a passive control group, we cannot estimate the absolute effect size, only the differences between conditions. In evaluating these, it is important to consider that most educational interventions have small effect sizes, the mean of 141 interventions was recently estimated to be $0.06^{41,42}$. However, small effects are important if they have a repeated impact, such as improved learning processes over time ${ }^{31}$.

Another limitation of this study is that we cannot evaluate how effective cognitive training is relative to mathematical training alone because all children had the same proportion of math training. It is likely that for any given test, for example of addition or geometry, training on that particular skill is the most time-effective way to improve test results. However, this study offers a proof of principle that spatial cognitive training transfers to academic abilities. Given the wide range of areas associated with spatial cognition - including not only other fields of mathematics, but also science, technology, and engineering - it is possible that training transfers to multiple areas, which should be included in any calculation by teachers and policymakers of how time-efficient spatial training is relative to training for a particular test.

\section{Methods}

\section{Implementation and inclusion criteria}

Data was collected in collaboration with the non-profit foundation Cognition Matters to implement our training plans using their freely available app 'Vektor' (https://cognitionmatters.org/). Vektor is an adaptive cognitive training app primarily aimed at improving school performance in mathematics. Previous research has shown the efficacy of 'Vektor' to improve mathematics in children ${ }^{34}$. There was no active recruitment or advertising for this study. Educators signed up their classes entirely by their own volition, including agreeing on data storage and children's data was automatically anonymized. The study design was approved by the regional Ethical Committee. Educators choose the amount of training per day, either 20 or 33 minutes, and the app automatically logged out after the prescribed amount to time.

Children were only included if they completed 36 days of training (corresponding to the mathematical tests in week 7) and if they were between the ages of six and eight (see SI Fig. 8 for attrition). The app would automatically randomize each account to one of the training plans, assuring that schools with differing demographics would have equal percentages of students in each training plan. Data were collected remotely over five semesters (Aug 2017 - Dec 2019), each semester was coded as a cohort to be used as a covariate. In August 2018, we added two training plans (Rotation heavy \& Rotation/NVR) and removed the NVR plan (SI Fig. 2 \& SI Table 1).

Training tasks and plans 
For the first five days, every child completed an identical proportion of training tasks: working memory grid, number line, non-verbal reasoning (NVR) and 2D mental rotation (SI Fig 1 for task examples). Each task had a certain portion of 'guided' trials to help teach the children how the task functioned. Working memory grid is a type of vs-WM training in which the subject is presented with a sequence of dots in different locations on a grid and needs to accurately reproduce the sequence by touching the screen ${ }^{43}$. The app automatically adjusts difficulty by increasing the span of items. Number line training starts simply by instructing the child to use their index fingers to drag the number line to the correct position corresponding to an Arabic numeral. The difficulty is initially moderated by removing spatial cues (e.g., ticks on the number line) it than progresses to incorporate mathematical problems (e.g., addition, subtraction with gradually larger numbers, then introducing negative numbers, decimals and fractions). Ten pals, another number line task, consist of bars on the right side of the screen that are partially filled number lines, the subject is than instructed to pull the correct bar (i.e., the one with the right number of units to fit) from multiple options on the right side ${ }^{44}$. The difficultly was moderated by bar length, removal of bars for Arabic numerals and increasing the sum from 5 to 10 and then 15. 2D mental rotation consisted of mental rotation tasks where difficulty was increased by increasing the angle of the required rotation and the complexity of the objects being rotated, similar to other rotation training in the literature ${ }^{28}$. The NVR task consisted of a sequential ordering tasks in which subjects viewed a sequence of tiles with spatial patterns and had to choose the correct image to fill the blank in the sequence ${ }^{45}$. Difficulty was increased by introducing additional stimulus dimensions (colors, shapes, number of dots) on which the stimuli should be compared.

After the first week, children were randomly divided into 5 training plans. The default training plan included only vs-WM and mathematical training, this plan is identical to the one showing the highest mean gains in Nemmi et al., 2016. Due to ethical considerations, we did not want to deviate too far from this previous design. The experimental training plans substituted vsWM for the domain of interest; see SI Table 1 for proportions.

\section{Testing tasks}

Testing was self-administered through the application and appeared on specific days: the first testing session was delivered on the $3^{\text {rd }}$ and $4^{\text {th }}$ days of training, the second on the $25^{\text {th }}$ and $26^{\text {th }}$ days of training and the final session on the $35^{\text {th }}$ and $36^{\text {th }}$ days of training. Addition and subtraction tests appeared on the first day per testing session while a number comparison task appears the following day. For the addition and subtraction tasks, children responded with onscreen buttons (0-9). The test finished if a trial was not completed within 60-seconds or if 3 consecutive errors were made. The sum of correct responses was used as the outcome measure for both tests. The number comparison task presented two single-digit Arabic numerals on either side of the screen and the children had to respond by tapping on the larger number. The outcome measure was the mean response time of correct trials.

As expected in this age range, the subtraction and addition tests were not normally distributed, due to a large number of zero responses in the first test (SI Fig. 4a). Yet the third test, mean reaction time of correct number comparison trials, was normally distributed. Around two percent of the number comparison test data was missing and therefore imputed, this represented under $1 \%$ of total test data. 


\section{Validity of online mathematical testing}

To test the validity of the online tasks, we used data from two schools gathered over the course of a year. The first study consisted of 46 preschool-age children, while the second consisted of 60 children ( 36 of which were in first grade). Ethical approval was granted by a regional Ethics Committee. A trained experimenter individually administered an addition, subtraction and a WISC verbal arithmetic test. The online tests are identical to the ones used in this study. The time between experimenter administered and online tests was a maximum of four weeks. Both school and grade were residualized from the mathematical tests. We then fit exploratory factor analyses' individually for experimenter-administered and online tests, using the 'fa' function from the R package 'psych'46,47.

Following the extraction of factor scores, we used a Pearson correlation to determine the consistency between math tests administered in person and those given online. It should be noted that these factors will not represent exactly the same underlying concept (i.e., lacking configural invariance), as number comparison is being substituted by the WISC verbal arithmetic test.

\section{Confirmatory factor analyses (CFA)}

A CFA was used in which the three self-administered tests were combined into a latent mathematical factor for each time point, this was done to increase power by removing measurement error. Correlated error variance was modeled between time for each task. For model estimation we used full information maximum likelihood and a robust maximum likelihood estimator with a Yuan-Bentler scaled test statistic from the R package Lavaan ${ }^{48}$. Missing follow-up behavioral data was imputed under the assumption of missing at random ${ }^{49}$. We assessed model fit using the comparative fit index (CFI; fit $>0.95)$ and the root mean square error of approximation (RMSEA; fit $<.08)^{50}$.

The model was then tested for longitudinal measurement invariance. This is a necessary step to ensure the latent factors represent the same concept throughout time. This procedure is a series of increasingly strict tests, 1) the loadings are constrained to be equal through time (weak invariance); 2) the intercepts are constrained to be equal between the time points (strong invariance); and 3) also the error variances of each of the tests are constrained to be equal through time (strict invariance; SI Fig. 5). The end result is four models: the baseline model (i.e. configural invariance) in which the same tests are used through time, weak, strong and strict invariant models. A series of model comparisons, in ascending order of strictness, are run to determine longitudinal measurement invariance. Regrettably, there is no consensus in the literature about which fit indices to use or the cutoff values therefore we decided to include a variety of fit indices in SI Table $3^{51}$. Due to our large sample size, we decided to test invariance via a change of $\geq-.010$ in CFI, supplemented by a change of $\geq .015$ in RMSEA ${ }^{52}$.

\section{Linear mixed-effects modeling}

Factor scores were estimated from a longitudinal strict measurement invariant CFA to be used as the dependent variable in a mixed-effects models using maximum likelihood estimation with the R package lme $4^{53}$. This modeling strategy was chosen over a latent growth curve model mainly due to the numerous categorical covariates along with the ability to easily compare 
coefficients. Covariates were added step-wise using AIC model comparison. An AIC difference of 8 was considered meaningful for model fit. The 'emmeans' $\mathrm{R}$ package was used to estimate marginal means and test contrasts ${ }^{53}$.

All models, except the intercept only model, were fit with a correlated random intercept and slope per subject per test week. Test week was coded using the number of weeks in-between tests (i.e., $\mathrm{t} 1=0, \mathrm{t} 2=4, \mathrm{t} 3=6$ ). Models were built hierarchically, by adding fixed effects for test week, age bracket, cohort and training time. To test the effect of training time we added an interaction of training time with test week.

\section{Effect of different cognitive training}

We took three complementary approaches to determine the effect of different types of training. The first two built upon earlier models, while for the third we took a different strategy by extracted random slopes and intercepts. For the first approach, we added training plans (categorically dummy coded) building upon the aforementioned model (i.e., with an interaction of training time with test week). A model with training plans interacting with test week was compared against one with only the fixed effects and a more parsimonious model without training plans included.

In the second approach, we added terms for the amount of rotation (as a combination of 2D mental rotation and tangram) and the amount of NVR training. The amount of rotation or NVR training did not take into consideration if subjects had trained 20 or 33 minutes, as this variance is already accounted for in a simpler model (via the interaction of test week with training time). A model with rotation and NVR terms both interacting with test week was compared against one with only fixed effects, and again a more parsimonious model. We refer the reader to AIC differences in model comparisons to draw conclusions, yet when we do report p-values from mixed-effects models these are derived using the Satterthwaite's degrees of freedom method.

The third method was used for two reasons 1) to estimate the amount of effect from each spatial task and 2) to check that treating 2D mental rotation and tangram as similar tasks holds. To accomplish this goal, we extracted random intercepts from subjects and random slopes of test week from a model with fixed effects for test week, age bracket, cohort and test time. We then coded four tasks (vs-WM, NVR, 2D mental rotation, tangram) based on the daily minutes of training of each of them, taking into consideration whether subjects training 20 or 33 minutes. These were entered into a linear model predicting children's random slopes (standardized with a mean $=0 \& \mathrm{SD}=1$ ) while correcting for their baseline mathematics (random intercepts).

\section{Predicting improvement based on performance in the first week of training}

Since all subjects completed identical cognitive training within the first week, this allowed us to extract training indices from four tasks; vs-WM, Number line, Rotation and NVR. For performance measures, we used the mean level of correct trials and the accuracy (i.e., percentage of correct trials).

This resulted in a total of eight cognitive training predictors that were entered in a linear model predicting children's improvement. For our measure of interindividual improvement we extracted children's coefficients for test week (i.e., their random slopes) from a mixed-effects model with fixed effects for test week, age bracket, cohort and training time. We controlled for 
subject's baseline mathematics (i.e., their random intercepts), to see what predicts mathematical improvement independent of baseline abilities.

Lastly, we carried out three analyses in which we examined the interactions between the amount of different training (i.e, Rotation, NVR \& vs-WM) and baseline training indices to predict improvement. Similar to an earlier analysis, we coded amounts of training taking into consideration if children completed 20 or 33 mins per day. This analysis was based on the rationale that subjects struggling in one cognitive domain might benefit from extra training in this domain which then could transfer to mathematical improvement. We used FDR-correction to control our type 1 error rate in all prediction-based analyses.

\section{Data Availability}

The data and the code to replicate the main analysis (i.e., mixed effects model) will become available after publication at https://github.com/njudd/spatialcognition. Data for baseline characteristics and graphs in this study are available upon request from the corresponding author.

\section{Citations}

1. Wai, J., Lubinski, D. \& Benbow, C. P. Spatial ability for STEM domains: Aligning over 50 years of cumulative psychological knowledge solidifies its importance. J. Educ. Psychol. 101, 817-835 (2009).

2. Hawes, Z. \& Ansari, D. What explains the relationship between spatial and mathematical skills? A review of evidence from brain and behavior. Psychon. Bull. Rev. (2020) doi:10.3758/s13423-019-01694-7.

3. Mix, K. S. et al. Separate but correlated: The latent structure of space and mathematics across development. J. Exp. Psychol. Gen. 145, 1206-1227 (2016).

4. Peng, P., Namkung, J., Barnes, M. \& Sun, C. A meta-analysis of mathematics and working memory: Moderating effects of working memory domain, type of mathematics skill, and sample characteristics. J. Educ. Psychol. 108, 455-473 (2016).

5. Gathercole, S. E. \& Brown, L. Working memory assessments at school entry as longitudinal predictors of National Curriculum attainment levels. Educational and Child (2003). 
6. Geary, D. C. Cognitive predictors of achievement growth in mathematics: a 5-year longitudinal study. Dev. Psychol. 47, 1539-1552 (2011).

7. Dillon, M. R., Kannan, H., Dean, J. T., Spelke, E. S. \& Duflo, E. Cognitive science in the field: A preschool intervention durably enhances intuitive but not formal mathematics. Science 357, 47-55 (2017).

8. Newcombe, N. Harnessing spatial thinking to support stem learning. (2017).

9. Stieff, M. \& Uttal, D. How Much Can Spatial Training Improve STEM Achievement? Educ. Psychol. Rev. 27, 607-615 (2015).

10. Ontario Ministry of Education. Paying Attention to Spatial Reasoning, K-12 Support Document for Paying Attention to Mathematics Education. (2014).

11. Lohman, D. F. Spatial abilities as traits, processes, and knowledge. in Advances in the psychology of human intelligence, Vol (ed. Sternberg, R. J.) vol. 4 181-248 (Lawrence Erlbaum Associates, Inc, xv, 1988).

12. Carpenter, P. A. \& Just, M. A. Spatial ability: AN information processing approach to psychometrics. in Advances in the psychology fo human intelligence. (ed. Stenberg, R. J.) 221-252 (Hillsdale, NJ: Erlbaum, 1986).

13. Cheng, Y.-L. \& Mix, K. S. Spatial Training Improves Children's Mathematics Ability. J. Cogn. Dev. 15, 2-11 (2014).

14. Hawes, Z., Moss, J., Caswell, B., Naqvi, S. \& MacKinnon, S. Enhancing Children's Spatial and Numerical Skills through a Dynamic Spatial Approach to Early Geometry Instruction: Effects of a 32-Week Intervention. Cogn. Instr. 35, 236-264 (2017). 
15. Lowrie, T., Logan, T. \& Hegarty, M. The Influence of Spatial Visualization Training on Students' Spatial Reasoning and Mathematics Performance. J. Cogn. Dev. 20, 729-751 (2019).

16. Hawes, Z., Moss, J., Caswell, B. \& Poliszczuk, D. Effects of mental rotation training on children's spatial and mathematics performance: A randomized controlled study. Trends in Neuroscience and Education 4, 60-68 (2015).

17. Cornu, V., Schiltz, C., Pazouki, T. \& Martin, R. Training early visuo-spatial abilities: A controlled classroom-based intervention study. Appl. Dev. Sci. 1-21 (2017).

18. Rodán, A., Gimeno, P., Elosúa, M. R., Montoro, P. R. \& Contreras, M. J. Boys and girls gain in spatial, but not in mathematical ability after mental rotation training in primary education. Learn. Individ. Differ. 70, 1-11 (2019).

19. Wright, H. et al. Improving working memory. https://westminsterresearch.westminster.ac.uk/download/1d07359f1fda308387fc3679b914d ac0eb89947b618f7f68f999a6f87793bfba/1174522/Working\%20Memory.pdf (2019).

20. Berger, Eva M. and Fehr, Ernst and Hermes, Henning and Schunk, Daniel and Winkel, Kirsten, The Impact of Working Memory Training on Children's Cognitive and Noncognitive Skills (June 9, 2020). NHH Dept. of Economics Discussion Paper No. 09/2020, Available at SSRN: $\underline{\text { https://ssrn.com/abstract=3622985 }}$ or http://dx.doi.org/10.2139/ssrn.3622985

21. Bergman-Nutley, S. \& Klingberg, T. Effect of working memory training on working memory, arithmetic and following instructions. Psychol. Res. 78, 869-877 (2014). 
22. Roberts, G. et al. Academic Outcomes 2 Years After Working Memory Training for Children With Low Working Memory: A Randomized Clinical Trial. JAMA Pediatr. 170, e154568 (2016)

23. Schwaighofer, M., Fischer, F. \& Bühner, M. Does Working Memory Training Transfer? A Meta-Analysis Including Training Conditions as Moderators. Educ. Psychol. 50, 138-166 (2015).

24. Simons, D. J. et al. Do "Brain-Training" Programs Work? Psychol. Sci. Public Interest 17, 103-186 (2016).

25. Francis, G. Too good to be true: publication bias in two prominent studies from experimental psychology. Psychon. Bull. Rev. 19, 151-156 (2012).

26. Shawn Green, C. et al. Improving Methodological Standards in Behavioral Interventions for Cognitive Enhancement. Journal of Cognitive Enhancement 3, 2-29 (2019).

27. Mackintosh, N. \& Mackintosh, N. J. IQ and Human Intelligence. (OUP Oxford, 2011).

28. Bergman Nutley, S. et al. Gains in fluid intelligence after training non-verbal reasoning in 4-year-old children: a controlled, randomized study. Dev. Sci. 14, 591-601 (2011).

29. Klauer, K. J. \& Phye, G. D. Inductive Reasoning: A Training Approach. Rev. Educ. Res. 78, 85-123 (2008).

30. Mackey, A. P., Hill, S. S., Stone, S. I. \& Bunge, S. A. Differential effects of reasoning and speed training in children. Dev. Sci. 14, 582-590 (2011).

31. Nemmi, F. et al. Behavior and neuroimaging at baseline predict individual response to combined mathematical and working memory training in children. Dev. Cogn. Neurosci. 20, 43-51 (2016). 
32. Fischer, U., Moeller, K., Bientzle, M., Cress, U. \& Nuerk, H.-C. Sensori-motor spatial training of number magnitude representation. Psychon. Bull. Rev. 18, 177-183 (2011).

33. Outhwaite, L. A., Faulder, M., Gulliford, A. \& Pitchford, N. J. Raising Early Achievement in Math With Interactive Apps: A Randomized Control Trial. J. Educ. Psychol. 111, 284298 (2019).

34. Klingberg, T. et al. Computerized training of working memory in children with ADHD--a randomized, controlled trial. J. Am. Acad. Child Adolesc. Psychiatry 44, 177-186 (2005).

35. Jaeggi, S. M., Buschkuehl, M., Jonides, J. \& Perrig, W. J. Improving fluid intelligence with training on working memory. Proc. Natl. Acad. Sci. U. S. A. 105, 6829-6833 (2008).

36. Schmiedek, F., Lövdén, M. \& Lindenberger, U. Hundred Days of Cognitive Training Enhance Broad Cognitive Abilities in Adulthood: Findings from the COGITO Study. Front. Aging Neurosci. 2, (2010).

37. Roid, G. H. \& Miller, L. J. Leiter International Performance Scale-Revised: Examiner's Manual. Wood Dale, IL: Stoelting (1997).

38. Mix, K. S. Why Are Spatial Skill and Mathematics Related? Child Dev. Perspect. 13, 121126 (2019).

39. Lortie-Forgues, H. \& Inglis, M. Rigorous Large-Scale Educational RCTs Are Often Uninformative: Should We Be Concerned? Educ. Res. 48, 158-166 (2019).

40. Bloom, H. S., Hill, C. J., Black, A. R. \& Lipsey, M. W. Performance Trajectories and Performance Gaps as Achievement Effect-Size Benchmarks for Educational Interventions. J. Res. Educ. Eff. 1, 289-328 (2008).

41. Abelson, R. P. A variance explanation paradox: When a little is a lot. Psychological Bulletin vol. 97 129-133 (1985). 
42. Funder, D. C. \& Ozer, D. J. Evaluating Effect Size in Psychological Research: Sense and Nonsense. Advances in Methods and Practices in Psychological Science 2, 156-168 (2019).

43. Butterworth, B. \& Kovas, Y. Understanding neurocognitive developmental disorders can improve education for all. Science 340, 300-305 (2013).

44. Uttal, D. H. et al. The malleability of spatial skills: a meta-analysis of training studies. Psychol. Bull. 139, 352-402 (2013).

45. Revelle, W. psych: Procedures for Psychological, Psychometric, and Personality Research. (2019).

46. R Core Team. R: A Language and Environment for Statistical Computing. (2014).

47. Rosseel, Y. Lavaan: An R package for structural equation modeling and more. Version 0.5-12 (BETA). J. Stat. Softw. 48, 1-36 (2012).

48. Enders, C. K. \& Bandalos, D. L. The Relative Performance of Full Information Maximum Likelihood Estimation for Missing Data in Structural Equation Models. Struct. Equ. Modeling 8, 430-457 (2001).

49. Hu, L. \& Bentler, P. M. Cutoff criteria for fit indexes in covariance structure analysis: Conventional criteria versus new alternatives. Struct. Equ. Modeling 6, 1-55 (1999).

50. Putnick, D. L. \& Bornstein, M. H. Measurement Invariance Conventions and Reporting: The State of the Art and Future Directions for Psychological Research. Dev. Rev. 41, 71-90 (2016).

51. Chen, F. F. Sensitivity of Goodness of Fit Indexes to Lack of Measurement Invariance. Struct. Equ. Modeling 14, 464-504 (2007).

52. Bates, D., Mächler, M., Bolker, B. \& Walker, S. Fitting Linear Mixed-Effects Models Using lme4. Journal of Statistical Software vol. 67 1-48 (2015). 
53. Lenth, R. emmeans: Estimated Marginal Means, aka Least-Squares Means. (2019).

\section{Acknowledgements}

We would like to acknowledge Dr. Rita Almeida, David Sjölander, Jonas Beckeman, Dr. Bruno Sauce and Dr. Dawei Zhang for their extensive help in various aspects of the study along with Dr. Laura Outhwaite, Dr. Pekka Räsänen and Dr. Ola Helenius for reviewing a draft of the manuscript. The work was supported by contributions from Mattias and Sonia Westman, along with funding from The Swedish Medical Research Foundation.

\section{Author contributions}

Both authors contributed equally in all aspects of the study.

\section{Competing interests}

The authors declare no competing interests. 


\section{Supplementary information}

Training spatial cognition enhances mathematical learning - a randomized study in 17.000 children

Nicholas Judd \& Torkel Klingberg 


\section{Supplementary Figures}

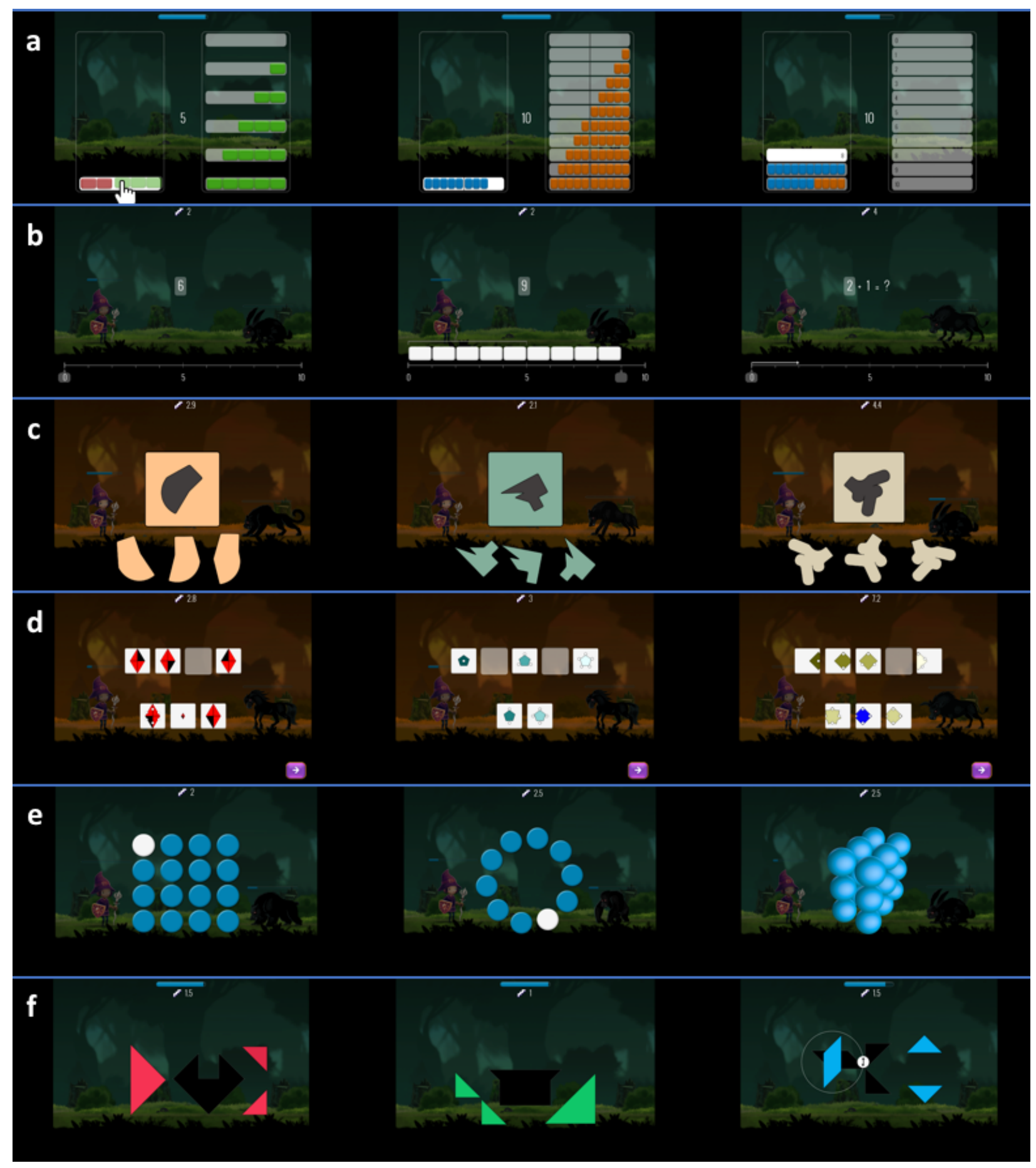

Sup. Figure 1 a) Npals another common number line task and b) the numberline task mentioned in text $c$ ) shows the 2D mental rotation task $\boldsymbol{d}$ ) shows the NVR task $\boldsymbol{e}$ ) vs-WM tasks, the first is the most common "WM grid" task which was used in text $f$ ) shows the Tangram task, we conceptually classify Tangram and 2D mental rotation as "rotation tasks" as both heavily tax rotation abelites. 


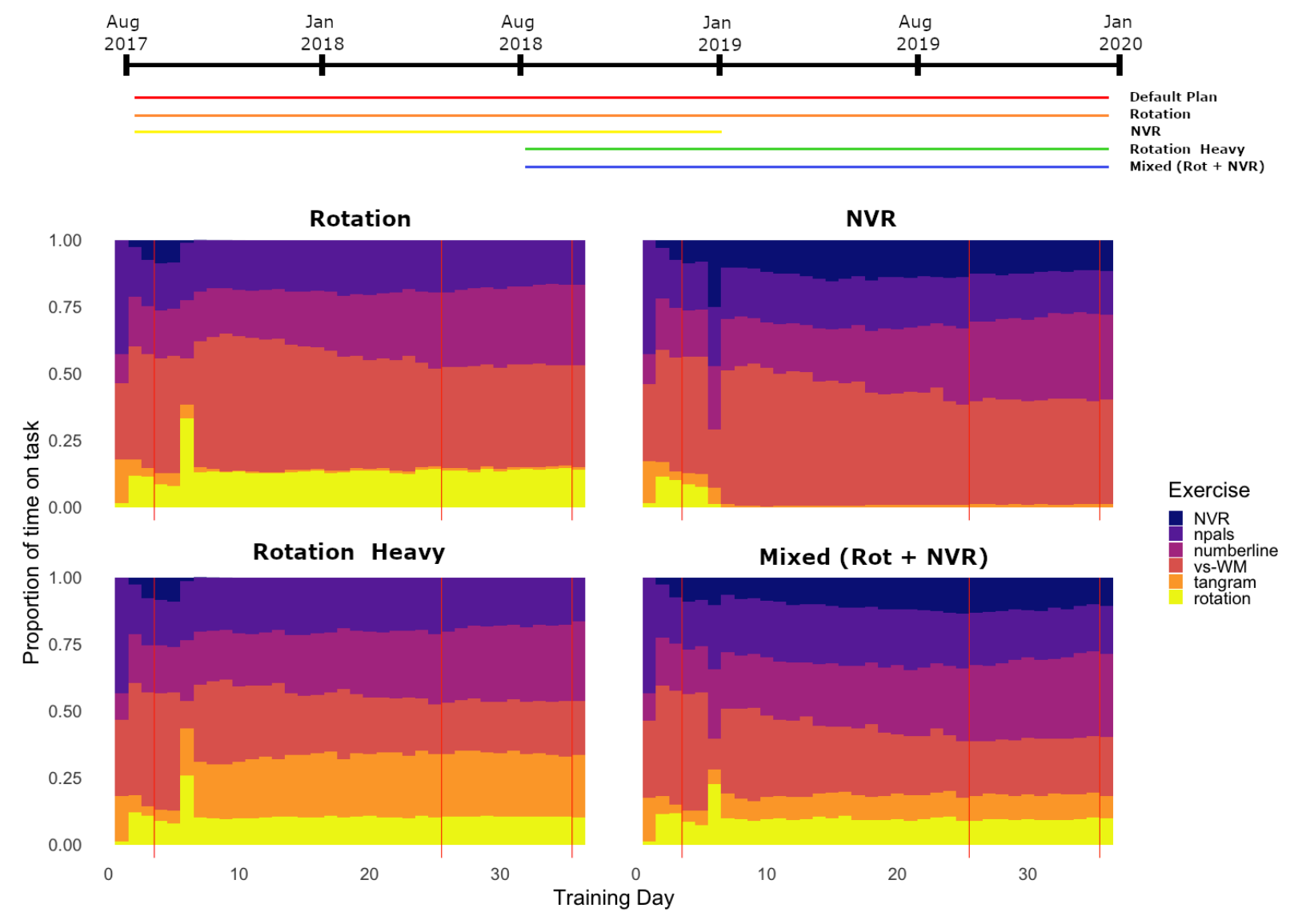

Supplementary Figure 2 Illustration of the training plans and the time over which they elapsed (this is a rough approximation; SI Table 1 supersedes this graph). Note each of the four experimental training plans have identical proportions of tasks within the first week. Following the fifth day subjects are randomly allocated into each training plan. Red lines denote the time of the mathematic tests. 
A
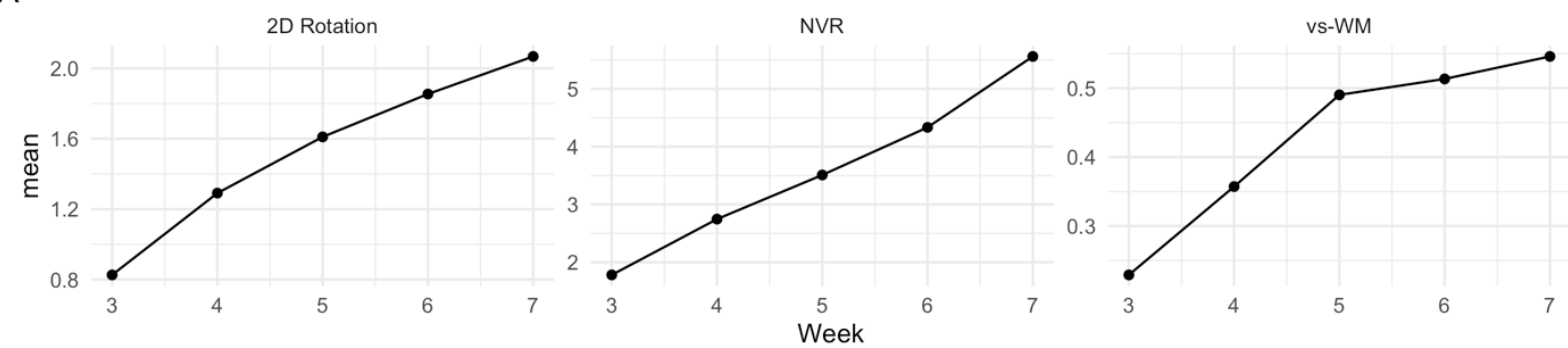

B

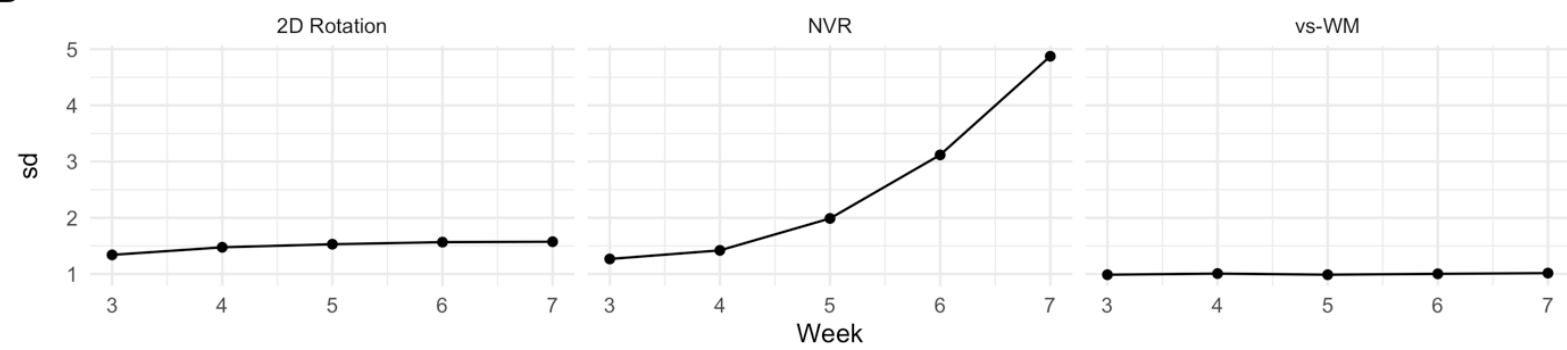

Sup Figure 3 Standardized training curves based on the mean and standard deviation from the 2nd week (which is the first week that children go into a specific training plan). A) Mean level; B) Standard deviation. 

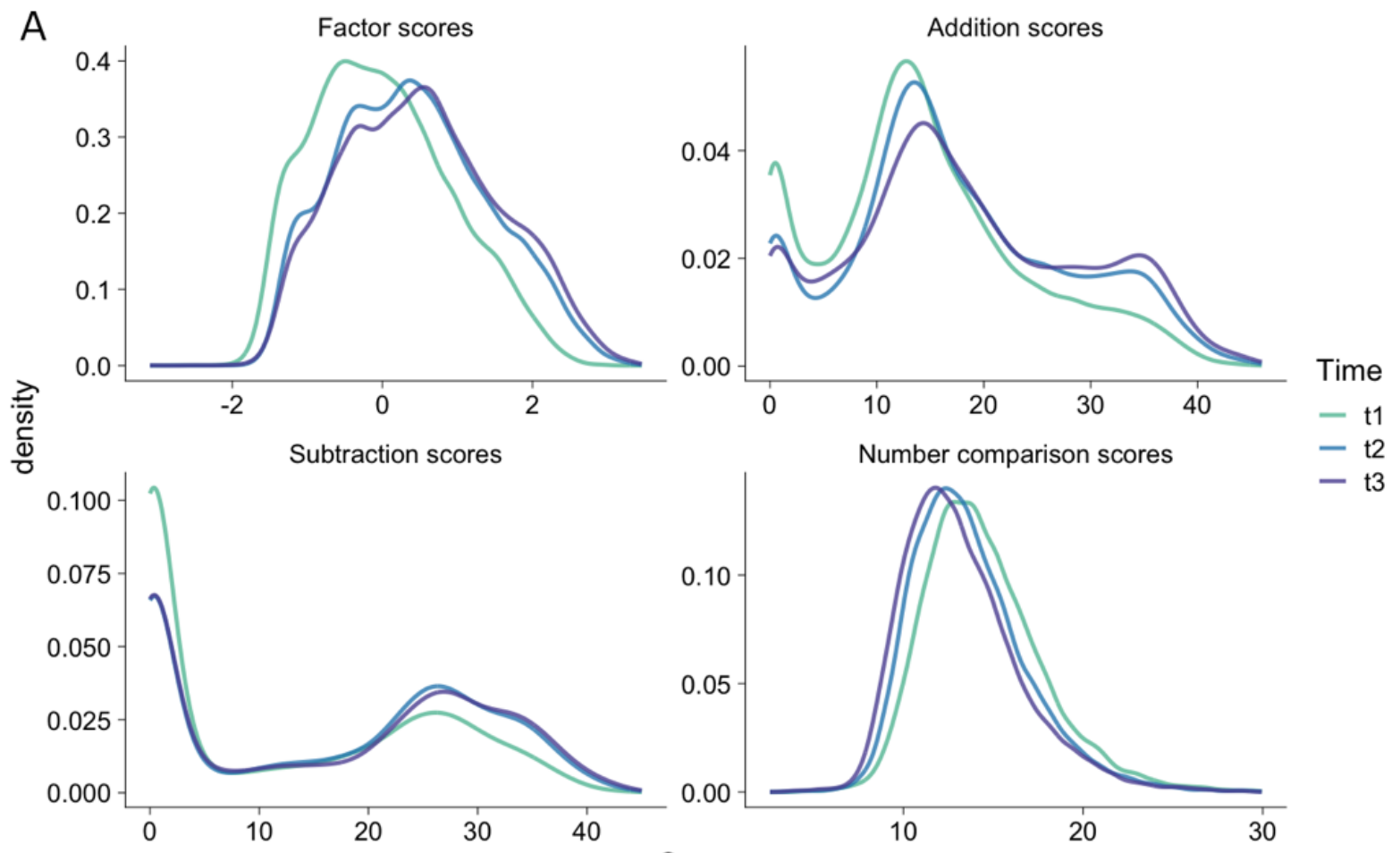

B

\section{Scores}
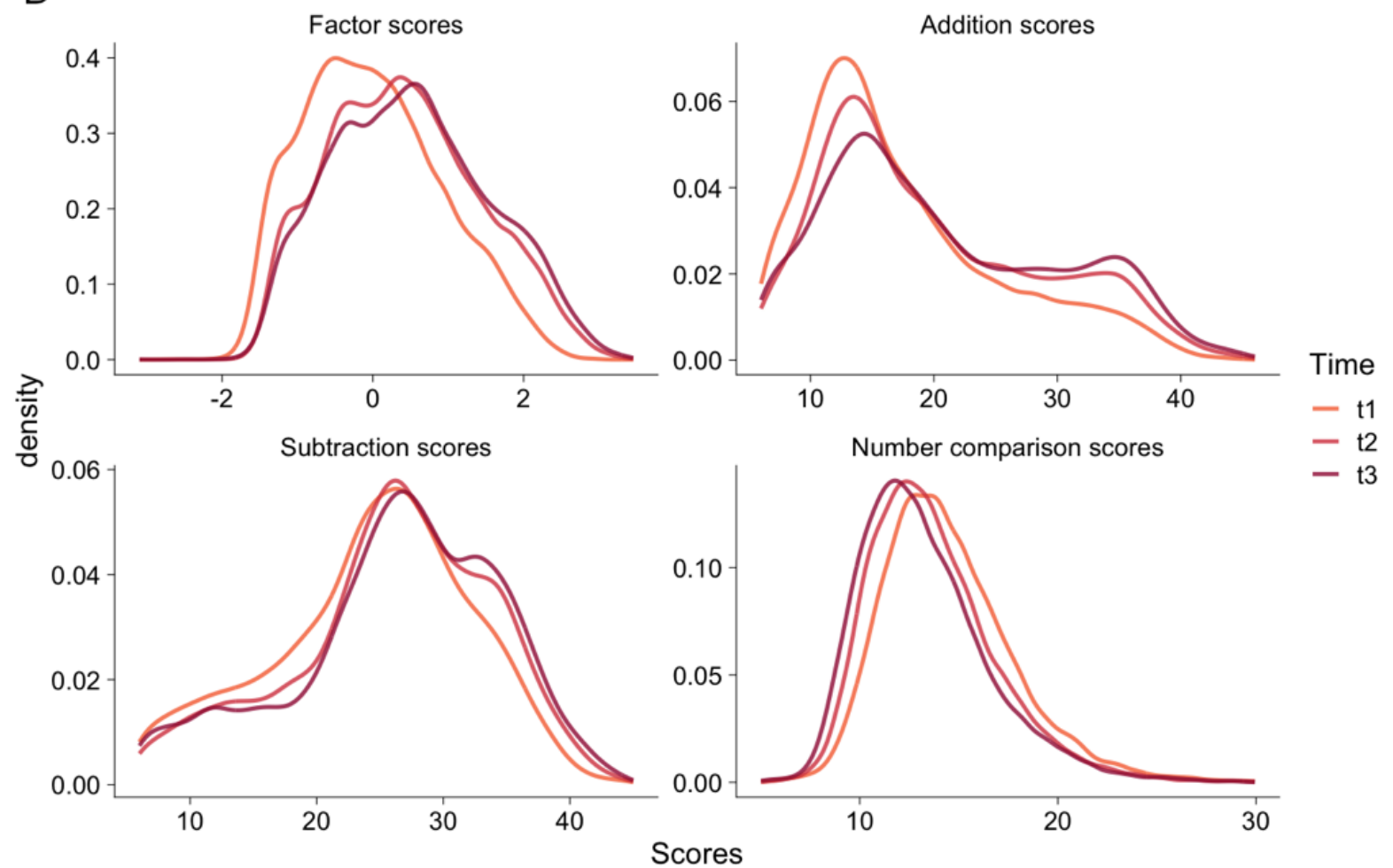

Sup. Figure 4 A) Density plots of predicted factor scores over test occasions and the tests that were used to make the factor score. $\boldsymbol{B})$ scores less than 5 are excluded for plotting purposes. 


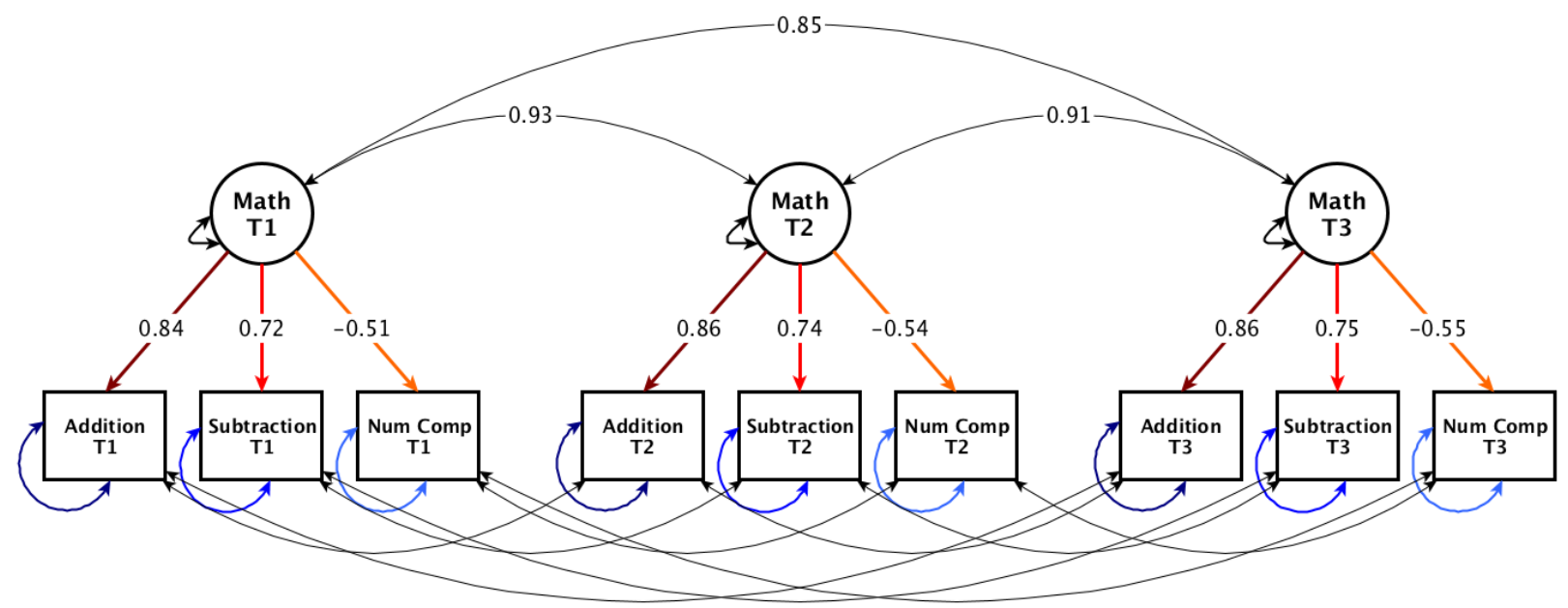

Supplementary Figure 5 Path diagram of a strict measurement invariant CFA for the mathematical tests. Loadings and covariances reported are standardized. Squares represent observed variables (i.e., math tests) while circles represent the latent mathematical factor for each test occasion. Single headed arrows denote regressions while double headed arrows represent covariances or error. 


\begin{tabular}{|c|c|c|c|c|c|c|c|}
\hline O_fa & & & & & & & \\
\hline 0.7 & $P_{\text {_fa }}$ & & & & & & \\
\hline 0.98 & 0.67 & O_add & & & & & \\
\hline 0.68 & 0.57 & 0.56 & O_sub & & & & \\
\hline-0.59 & -0.4 & -0.49 & -0.34 & O_num & & & \\
\hline 0.55 & 0.82 & 0.55 & 0.42 & $=0.23$ & P_verb & & \\
\hline 0.57 & 0.8 & 0.56 & 0.35 & -0.37 & 0.47 & $P_{\text {_add }}$ & \\
\hline 0.55 & 0.78 & 0.49 & 0.6 & -0.37 & 0.46 & 0.44 & P_sub \\
\hline
\end{tabular}

Supplementary Figure 6 Pearson correlation plot of online ("O"; $\mathrm{O} \_$add = Addition, O_sub = Subtraction, O_num = Number

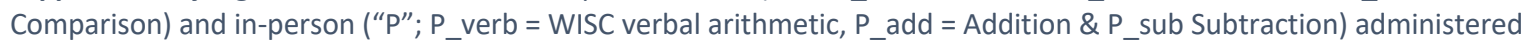
mathematical tasks, all correlations are significant. Data $(n=106)$ was collected in two schools, one of which had preschoolers and first graders. Both school and grade were residualized before extracting factor scores from an exploratory factor analysis for online tests (O_fa; loadings O_add $=.91$, O_sub $=.62$, O_num $=-.52$ ) and in-person tests ( $P_{-}$fa; loadings $P_{-}$verb $=.69, P_{-}$add $=.68 \& P_{-}$sub $\left.=.66\right)$. There was up to 4 weeks in-between online and in-person assessments. In a separate sample $(n=48)$, we tested the test-retest correlation of the in-person administered tasks after 5 weeks in a control group. Using the fa function from the psych package (keeping the loadings from the first timepoint) the test-retest correlation was .94. One point to note is that the online and in-person math factors differ by one task (WISC Verbal Arithmetic vs number comparison). 

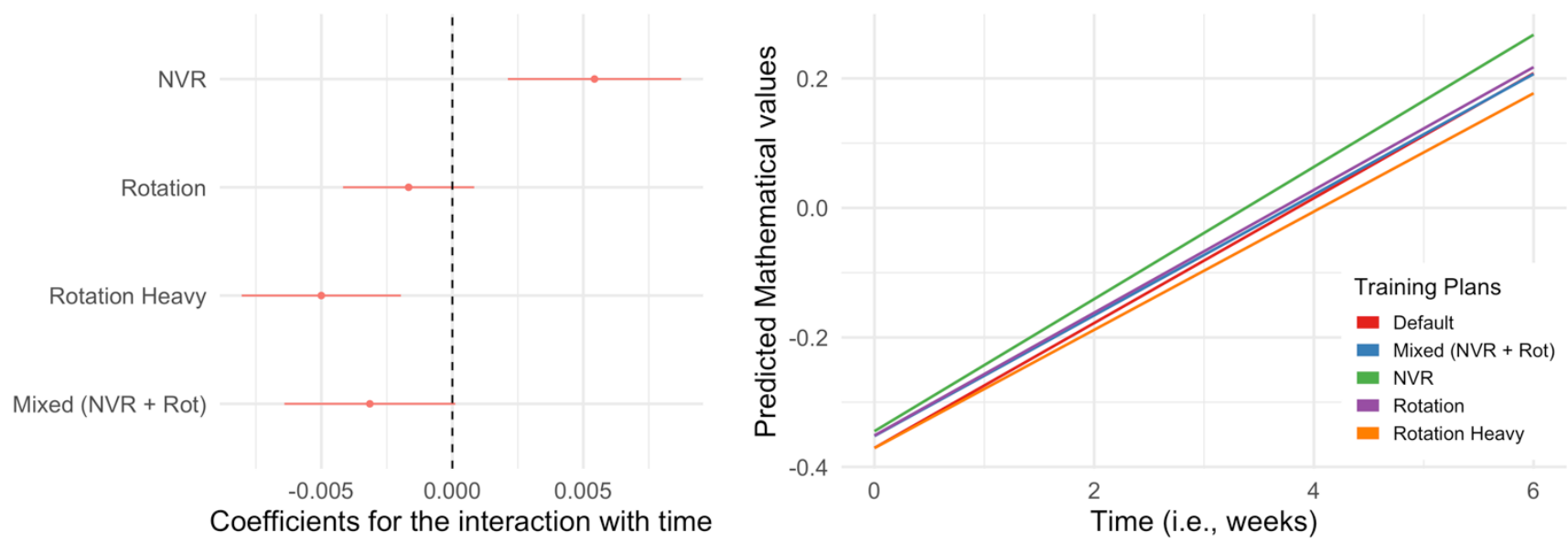

Sup Figure 7 Plots showing the effects of training plans using a linear mixed-effects model (model fit improvement of $20 \Delta$ AIC). A) First, a dot-plot with $95 \% \mathrm{Cl}$ 's showing the coefficients for the interaction of dummy coded training plans (intercept = default plan) with time for predicting mathematical factor scores. The second plot $\boldsymbol{B}$ ), shows the marginal effects of training plan assignment over the weeks (i.e., time) of training. A post-hoc analysis determined a significant difference $(b=.083, p<.05)$ by the end of training between the NVR group and the rotation heavy group. Both plots reflect the same model with fixed effects adjusting for age, cohort, amount of training and the interaction of amount of training with test week (see Sup Table 4c).

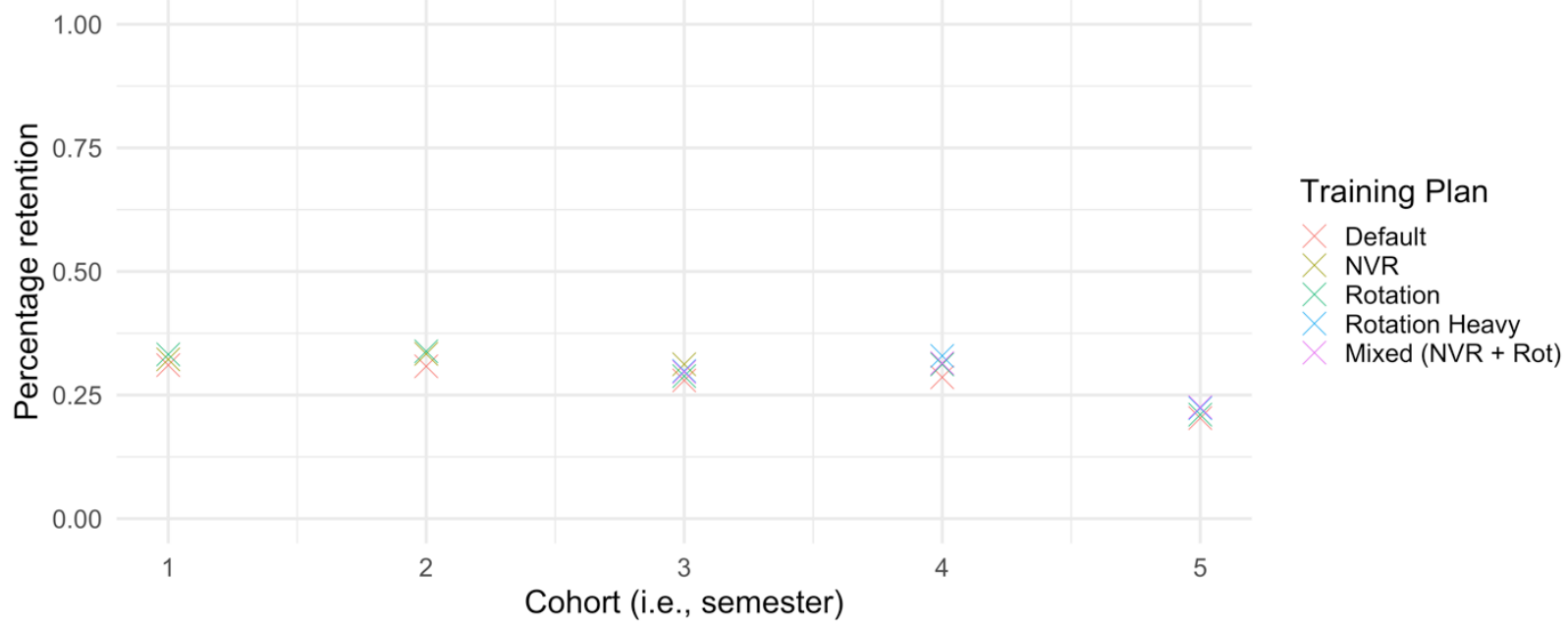

Sup Figure 8 Plot showing subject retention between training plans. Percentage retention was calculated based on the number of children that completed at least five days, as this reflects children before training plan randomization. Crucially, there was no systematic pattern of differential attrition between our best performing plan (i.e., NVR) and our worst (i.e., Rotation). 


\section{Supplementary Tables}

\section{Supplementary Table 1}

Percentage amounts of training tasks in each of the different training groups after the first week.

See plot 1 for an illustration.

\begin{tabular}{lcccccc}
\hline Training plan & n & \multicolumn{3}{c}{ Numberline vs-WM } & NVR & Rotation \\
\hline Default & 6,542 & $48.5 \%$ & $48.5 \%$ & $0 \%$ & $0 \%$ & $3 \%$ \\
NVR & 2,000 & $50 \%$ & $35 \%$ & $13.5 \%$ & $0 \%$ & $1.5 \%$ \\
Rotation & 4,520 & $50 \%$ & $35 \%$ & $0 \%$ & $13.5 \%$ & $1.5 \%$ \\
Rotation Heavy & 2,511 & $48.8 \%$ & $21.5 \%$ & $0 \%$ & $10 \%$ & $20 \%$ \\
Mixed (NVR + Rotation) & 2,075 & $48.8 \%$ & $21.5 \%$ & $10 \%$ & $10 \%$ & $10 \%$ \\
\hline
\end{tabular}

\section{Supplementary Table 2}

Descriptive table of test and corresponding factor data.

\begin{tabular}{lccccccc}
\hline \hline Statistic & N & Mean & St. Dev. & Min & Pctl(25) & Pctl(75) & Max \\
\hline t1_MathFactor & 17,648 & 0.00000 & 1.000 & -3.009 & -0.772 & 0.684 & 3.201 \\
t2_MathFactor & 17,648 & 0.452 & 1.087 & -2.214 & -0.388 & 1.206 & 3.730 \\
t3_MathFactor & 17,648 & 0.556 & 1.123 & -3.364 & -0.324 & 1.351 & 3.752 \\
t1_add & 17,648 & 14.516 & 9.491 & 0 & 8 & 20 & 46 \\
t2_add & 17,648 & 17.496 & 10.315 & 0 & 11 & 25 & 46 \\
t3_add & 17,648 & 18.331 & 10.868 & 0 & 11 & 27 & 46 \\
t1_sub & 17,648 & 12.476 & 13.123 & 0 & 0 & 25 & 45 \\
t2_sub & 17,648 & 17.357 & 13.568 & 0 & 1 & 29 & 45 \\
t3_sub & 17,648 & 17.419 & 13.925 & 0 & 1 & 29 & 45 \\
t1_num & 17,502 & 14.538 & 3.506 & 3.970 & 12.180 & 16.350 & 66.770 \\
t2_num & 17,273 & 13.709 & 3.318 & 3.670 & 11.410 & 15.450 & 49.860 \\
t3_num & 17,161 & 13.277 & 3.533 & 2.570 & 10.920 & 14.980 & 91.080 \\
\hline
\end{tabular}




\section{Supplementary Table 3}

Longitudinal Measurement Invariance fit measures.

\begin{tabular}{|c|c|c|c|c|}
\hline & configuralcorcov & weak & $\overline{\text { strong }}$ & strict \\
\hline npar & 39.000 & 35.000 & 31.000 & 25.000 \\
\hline fmin & 0.028 & 0.034 & 0.049 & 0.074 \\
\hline chisq & 981.954 & 1211.553 & 1746.031 & 2626.102 \\
\hline df & 15.000 & 19.000 & 23.000 & 29.000 \\
\hline pvalue & 0.000 & 0.000 & 0.000 & 0.000 \\
\hline chisq.scaled & 866.234 & 1089.167 & 1476.132 & 1261.833 \\
\hline df.scaled & 15.000 & 19.000 & 23.000 & 29.000 \\
\hline pvalue.scaled & 0.000 & 0.000 & 0.000 & 0.000 \\
\hline chisq.scaling.factor & 1.134 & 1.112 & 1.183 & 2.081 \\
\hline baseline.chisq & 89777.605 & 89777.605 & 89777.605 & 89777.605 \\
\hline baseline.df & 36.000 & 36.000 & 36.000 & 36.000 \\
\hline baseline.pvalue & 0.000 & 0.000 & 0.000 & 0.000 \\
\hline baseline.chisq.scaled & 29761.757 & 29761.757 & 29761.757 & 29761.757 \\
\hline baseline.df.scaled & 36.000 & 36.000 & 36.000 & 36.000 \\
\hline baseline.pvalue.scaled & 0.000 & 0.000 & 0.000 & 0.000 \\
\hline baseline.chisq.scaling.factor & 3.017 & 3.017 & 3.017 & 3.017 \\
\hline cfi & 0.989 & 0.987 & 0.981 & 0.971 \\
\hline tli & 0.974 & 0.975 & 0.970 & 0.964 \\
\hline nnfi & 0.974 & 0.975 & 0.970 & 0.964 \\
\hline rfi & 0.974 & 0.974 & 0.970 & 0.964 \\
\hline nfi & 0.989 & 0.987 & 0.981 & 0.971 \\
\hline pnfi & 0.412 & 0.521 & 0.626 & 0.782 \\
\hline ifi & 0.989 & 0.987 & 0.981 & 0.971 \\
\hline rni & 0.989 & 0.987 & 0.981 & 0.971 \\
\hline cfi.scaled & 0.971 & 0.964 & 0.951 & 0.959 \\
\hline tli.scaled & 0.931 & 0.932 & 0.923 & 0.949 \\
\hline cfi.robust & 0.989 & 0.987 & 0.981 & 0.971 \\
\hline tli.robust & 0.974 & 0.975 & 0.970 & 0.964 \\
\hline nnfi.scaled & 0.931 & 0.932 & 0.923 & 0.949 \\
\hline nnfi.robust & 0.974 & 0.975 & 0.970 & 0.964 \\
\hline rfi.scaled & 0.930 & 0.931 & 0.922 & 0.947 \\
\hline nfi.scaled & 0.971 & 0.963 & 0.950 & 0.958 \\
\hline ifi.scaled & 0.971 & 0.964 & 0.951 & 0.959 \\
\hline rni.scaled & 0.971 & 0.964 & 0.951 & 0.959 \\
\hline rni.robust & 0.989 & 0.987 & 0.981 & 0.971 \\
\hline $\log 1$ & -504840.924 & -504955.723 & -505222.962 & -505662.998 \\
\hline unrestricted.logl & -504349.947 & -504349.947 & -504349.947 & -504349.947 \\
\hline aic & 1009759.847 & 1009981.447 & 1010507.924 & 1011375.995 \\
\hline
\end{tabular}




\begin{tabular}{lcccr}
\hline \hline & configuralcorcov & weak & strong & strict \\
\hline bic & 1010063.206 & 1010253.692 & 1010749.056 & 1011570.456 \\
ntotal & 17649.000 & 17649.000 & 17649.000 & 17649.000 \\
bic2 & 1009939.266 & 1010142.464 & 1010650.540 & 1011491.007 \\
scaling.factor.h1 & 2.727 & 2.727 & 2.727 & 2.727 \\
scaling.factor.h0 & 3.029 & 2.933 & 2.792 & 2.021 \\
rmsea & 0.060 & 0.060 & 0.065 & 0.071 \\
rmsea.ci.lower & 0.057 & 0.057 & 0.063 & 0.069 \\
rmsea.ci.upper & 0.064 & 0.063 & 0.068 & 0.074 \\
rmsea.pvalue & 0.000 & 0.000 & 0.000 & 0.000 \\
rmsea.scaled & 0.057 & 0.056 & 0.060 & 0.049 \\
rmsea.ci.lower.scaled & 0.054 & 0.054 & 0.057 & 0.047 \\
rmsea.ci.upper.scaled & 0.060 & 0.059 & 0.062 & 0.051 \\
rmsea.pvalue.scaled & 0.000 & 0.000 & 0.000 & 0.825 \\
rmsea.robust & 0.060 & 0.060 & 0.065 & 0.071 \\
rmsea.ci.lower.robust & 0.057 & 0.057 & 0.062 & 0.067 \\
rmsea.ci.upper.robust & 0.064 & 0.063 & 0.068 & 0.074 \\
rmr & 1.592 & 3.680 & 3.791 & 3.231 \\
rmr_nomean & 1.744 & 4.031 & 4.151 & 3.537 \\
srmr & 0.026 & 0.031 & 0.030 & 0.049 \\
srmr_bentler & 0.025 & 0.034 & 0.033 & 0.037 \\
srmr_bentler_nomean & 0.028 & 0.037 & 0.033 & 0.038 \\
crmr & 0.027 & 0.031 & 0.030 & 0.049 \\
crmr_nomean & 0.026 & 0.030 & 0.025 & 0.035 \\
srmr_mplus & 0.026 & 0.031 & 0.030 & 0.049 \\
srmr_mplus_nomean & 0.024 & 0.030 & 0.027 & 0.037 \\
\hline \hline
\end{tabular}




\section{Supplementary Table 4a}

Linear mixed effects models predicting factor scores

\begin{tabular}{|c|c|c|c|c|c|c|c|}
\hline & Intercept only & $\begin{array}{l}\text { Random } \\
\text { slope }\end{array}$ & $\begin{array}{l}\text { Fixed effect } \\
\text { test week }\end{array}$ & $\begin{array}{c}\text { Fixed effect } \\
\text { Age }\end{array}$ & $\begin{array}{c}\text { Fixed effect } \\
\text { Cohort }\end{array}$ & $\begin{array}{l}\text { Fixed effect } \\
\text { Training time }\end{array}$ & $\begin{array}{l}\text { Interaction of } \\
\text { training time } \\
\text { with test week }\end{array}$ \\
\hline Predictors & Estimates & Estimates & Estimates & Estimates & Estimates & Estimates & Estimates \\
\hline (Intercept) & $\begin{array}{c}0.336^{* * *} \\
(0.320-0.351)\end{array}$ & $\begin{array}{c}-0.126^{* * *} \\
(-0.141-- \\
0.111)\end{array}$ & $\begin{array}{c}0.017^{*} \\
(0.002-0.032)\end{array}$ & $\begin{array}{c}-0.344^{* * *} \\
(-0.362-- \\
0.327)\end{array}$ & $\begin{array}{c}-0.237^{* * *} \\
(-0.272-- \\
0.203)\end{array}$ & $\begin{array}{c}-0.360^{* * *} \\
(-0.399-- \\
0.322)\end{array}$ & $\begin{array}{c}-0.368^{* * *} \\
(-0.407-- \\
0.329)\end{array}$ \\
\hline Test week & & & $\begin{array}{c}0.096^{* * *} \\
(0.095-0.097)\end{array}$ & $\begin{array}{c}0.096^{* * *} \\
(0.095-0.097)\end{array}$ & $\begin{array}{c}0.096^{* * *} \\
(0.095-0.097)\end{array}$ & $\begin{array}{c}0.096^{* * *} \\
(0.095-0.097)\end{array}$ & $\begin{array}{c}0.089^{* * *} \\
(0.087-0.091)\end{array}$ \\
\hline age [_s 7-8] & & & & $\begin{array}{c}0.901^{* * *} \\
(0.874-0.928)\end{array}$ & $\begin{array}{c}0.892^{* * *} \\
(0.865-0.920)\end{array}$ & $\begin{array}{c}0.873^{* * *} \\
(0.845-0.900)\end{array}$ & $\begin{array}{c}0.873^{* * *} \\
(0.845-0.900)\end{array}$ \\
\hline cohort [c2] & & & & & $\begin{array}{c}-0.100^{* * *} \\
(-0.145-- \\
0.055)\end{array}$ & $\begin{array}{c}-0.088^{* * *} \\
(-0.133-- \\
0.044)\end{array}$ & $\begin{array}{c}-0.088^{* * *} \\
(-0.133-- \\
0.044)\end{array}$ \\
\hline cohort [c3] & & & & & $\begin{array}{c}-0.088^{* * *} \\
(-0.128-- \\
0.048)\end{array}$ & $\begin{array}{c}-0.085^{* * *} \\
(-0.125-- \\
0.045)\end{array}$ & $\begin{array}{c}-0.085^{* * *} \\
(-0.125-- \\
0.045)\end{array}$ \\
\hline cohort [c4] & & & & & $\begin{array}{c}-0.163^{* * *} \\
(-0.204-- \\
0.121)\end{array}$ & $\begin{array}{c}-0.144^{* * *} \\
(-0.186-- \\
0.103)\end{array}$ & $\begin{array}{c}-0.144^{* * *} \\
(-0.186-- \\
0.103)\end{array}$ \\
\hline
\end{tabular}


cohort [c5]

training_time

[33]

Test week *

training_time

[33]

\section{Random Effects}

\begin{tabular}{|c|c|c|c|c|c|c|c|}
\hline$\sigma^{2}$ & 0.14 & & 0.04 & 0.04 & 0.04 & 0.04 & 0.04 \\
\hline$\tau_{00}$ & 1.06 account_id & & 0.98 account_id & 0.79 account_id & 0.78 account_id & 0.77 account_id & 0.77 account_id \\
\hline$\tau_{11}$ & & & 0.00 account_id.time & 0.00 account_id.time & 0.00 account_id.time & 0.00 account_id.time & 0.00 account_id.time \\
\hline$\rho_{01}$ & & & 0.27 account_id & 0.30 account_id & 0.29 account_id & 0.28 account_id & 0.28 account_id \\
\hline $\mathrm{ICC}$ & 0.88 & & 0.97 & 0.96 & 0.96 & 0.96 & 0.96 \\
\hline $\mathrm{N}$ & 17648 account_id & $\begin{array}{l}17648 \\
\text { account_id }\end{array}$ & 17648 account_id & 17648 account_id & 17648 account_id & 17648 account_id & 17648 account_id \\
\hline Observations & 52944 & 52944 & 52944 & 52944 & 52944 & 52944 & 52944 \\
\hline $\begin{array}{l}\text { Marg. } \mathrm{R}^{2} / \\
\text { Cond. } \mathrm{R}^{2}\end{array}$ & $0.000 / 0.880$ & NA & $0.047 / 0.969$ & $0.210 / 0.969$ & $0.213 / 0.969$ & $0.220 / 0.969$ & $0.225 / 0.969$ \\
\hline AIC & 103319.298 & 87509.227 & 67654.835 & 63859.373 & 63793.226 & 63627.282 & 63540.653 \\
\hline log-Lik & -51656.649 & -43749.61 & -33821.417 & -31922.687 & -31885.613 & -31801.641 & -31757.326 \\
\hline
\end{tabular}

$$
\begin{array}{ccc}
-0.186^{* * *} & -0.181^{* * *} & -0.181^{* * *} \\
(-0.240-- & (-0.234-- & (-0.234-- \\
0.132) & 0.127) & 0.127)
\end{array}
$$$$
(0.159-0.215) \quad(0.171-0.227)
$$$$
0.010^{* * *}
$$$$
(0.008-0.012)
$$ 


\section{Supplementary Table 4b}

AIC model selection of final model (3-way interaction of time, rotation training and nvr training) and dummy coded plans

\begin{tabular}{lrrrrrrr}
\hline Modnames & K & AICc & Delta_AICc & ModelLik & AICcWt & LL & Cum.Wt \\
\hline Interactions for Rotation and NVR & 17 & 63519.48 & 0.000000 & 1.0000000 & 0.8018107 & -31742.73 & 0.8018107 \\
Interactions for training plans & 21 & 63522.27 & 2.795557 & 0.2471454 & 0.1981638 & -31740.13 & 0.9999746 \\
Interaction of training time (Default) & 13 & 63540.66 & 21.180450 & 0.0000252 & 0.0000202 & -31757.33 & 0.9999947 \\
Only fixed effects Rotation and NVR & 15 & 63543.81 & 24.330985 & 0.0000052 & 0.0000042 & -31756.90 & 0.9999989 \\
Only fixed effects training plans & 17 & 63546.50 & 27.025624 & 0.0000014 & 0.0000011 & -31756.25 & 1.0000000 \\
\hline \hline
\end{tabular}




\section{Supplementary Table 4c}

Linear mixed effects models predicting factor scores for dummy coded training plans

\begin{tabular}{|c|c|c|c|}
\hline & $\begin{array}{l}\text { Interaction of training time } \\
\text { with test week }\end{array}$ & $\begin{array}{l}\text { Fixed effects for } \\
\text { training plans }\end{array}$ & $\begin{array}{l}\text { Interactions for } \\
\text { training plans }\end{array}$ \\
\hline Predictors & Estimates & Estimates & Estimates \\
\hline (Intercept) & $\begin{array}{c}-0.368^{* * *} \\
(-0.407--0.329)\end{array}$ & $\begin{array}{c}-0.380^{* * *} \\
(-0.423--0.337)\end{array}$ & $\begin{array}{c}-0.379^{* * *} \\
(-0.422--0.336)\end{array}$ \\
\hline cohort [c2] & $\begin{array}{c}-0.088^{* * *} \\
(-0.133--0.044)\end{array}$ & $\begin{array}{c}-0.088^{* * *} \\
(-0.133--0.044)\end{array}$ & $\begin{array}{c}-0.088^{* * *} \\
(-0.133--0.044)\end{array}$ \\
\hline cohort [c3] & $\begin{array}{c}-0.085^{* * *} \\
(-0.125--0.045)\end{array}$ & $\begin{array}{c}-0.085^{* * *} \\
(-0.127--0.042)\end{array}$ & $\begin{array}{c}-0.085^{* * *} \\
(-0.127--0.042)\end{array}$ \\
\hline cohort [c4] & $\begin{array}{c}-0.144^{* * *} \\
(-0.186--0.103)\end{array}$ & $\begin{array}{c}-0.144^{* * *} \\
(-0.190--0.099)\end{array}$ & $\begin{array}{c}-0.144^{* * *} \\
(-0.190--0.099)\end{array}$ \\
\hline cohort [c5] & $\begin{array}{c}-0.181^{* * *} \\
(-0.234--0.127)\end{array}$ & $\begin{array}{c}-0.180^{* * *} \\
(-0.237--0.124)\end{array}$ & $\begin{array}{c}-0.180^{* * *} \\
(-0.237--0.124)\end{array}$ \\
\hline Test week & $\begin{array}{c}0.089^{* * *} \\
(0.087-0.091)\end{array}$ & $\begin{array}{c}0.089^{* * *} \\
(0.087-0.091)\end{array}$ & $\begin{array}{c}0.090^{* * *} \\
(0.088-0.092)\end{array}$ \\
\hline age [_s 7-8] & $\begin{array}{c}0.873^{* * *} \\
(0.845-0.900)\end{array}$ & $\begin{array}{c}0.873^{* * *} \\
(0.846-0.900)\end{array}$ & $\begin{array}{c}0.873^{* * *} \\
(0.846-0.900)\end{array}$ \\
\hline training_time [33] & $\begin{array}{c}0.199^{* * *} \\
(0.171-0.227)\end{array}$ & $\begin{array}{c}0.199^{* * *} \\
(0.170-0.227)\end{array}$ & $\begin{array}{c}0.199^{* * *} \\
(0.170-0.227)\end{array}$ \\
\hline $\begin{array}{l}\text { Test week } * \\
\text { training_time [33] }\end{array}$ & $\begin{array}{c}0.010^{* * *} \\
(0.008-0.012)\end{array}$ & $\begin{array}{c}0.010^{* * *} \\
(0.008-0.012)\end{array}$ & $\begin{array}{c}0.010^{* * *} \\
(0.008-0.012)\end{array}$ \\
\hline Tplan_NVR & & $\begin{array}{c}0.019 \\
(-0.028-0.066)\end{array}$ & $\begin{array}{c}0.026 \\
(-0.022-0.073)\end{array}$ \\
\hline
\end{tabular}


Tplan_Rot

Tplan_RotHeavy

Tplan_mixed

Test week *

Tplan_NVR

Test week * Tplan_Rot

Test week *

Tplan_RotHeavy

Test week *

Tplan_mixed

\section{Random Effects}

$\sigma^{2}$

$\tau_{00} \quad 0.77$ account_id

$\tau_{11} \quad 0.00$ account_id.time

$\rho_{01} \quad 0.28$ account_id

ICC

$\mathrm{N} \quad 17648$ account_id

Observations

Marginal $\mathrm{R}^{2} /$ Con. $1 \mathrm{R}^{2}$

AIC

52944

$0.225 / 0.969$

63540.653
0.021
$(-0.013-0.055)$

0.006

$(-0.037-0.050)$

0.022

$(-0.024-0.069)$

0.019

$(-0.015-0.053)$$$
-0.000
$$

$(-0.044-0.044)$

0.019

$(-0.028-0.065)$

$0.005^{* *}$

$(0.002-0.009)$

$-0.002$

$(-0.004-0.001)$

$-0.005^{* *}$

$(-0.008--0.002)$

$-0.003$

$(-0.006-0.000)$

$\begin{array}{ll}0.04 & 0.04\end{array}$

0.77 account_id

0.77 account_id

0.00 account_id.time

0.00 account_id.time

0.28 account_id

0.28 account_id

0.96

0.96

17648 account_id

17648 account_id

52944

52944

0.225 / 0.969

$0.226 / 0.969$

63546.493

63522.257 
log-Likelihood

$-31757.326$

$-31756.247$

$* p<0.05 \quad * * p<0.01 \quad * * * p<0.001$ 


\section{Supplementary Table 4d}

Linear mixed effects models predicting factor scores for amounts of rotation and NVR

\begin{tabular}{|c|c|c|c|}
\hline Predictors & $\begin{array}{c}\text { Interaction of training } \\
\text { time with test week } \\
\text { Estimates }\end{array}$ & $\begin{array}{c}\text { Fixed effects for } \\
\text { Rotation and NVR } \\
\text { Estimates }\end{array}$ & $\begin{array}{c}\text { Interactions for } \\
\text { Rotation and NVR } \\
\text { Estimates }\end{array}$ \\
\hline (Intercept) & $\begin{array}{c}-0.368^{* * *} \\
(-0.407--0.329)\end{array}$ & $\begin{array}{c}-0.375^{* * *} \\
(-0.417--0.334)\end{array}$ & $\begin{array}{c}-0.373^{* * *} \\
(-0.414--0.331)\end{array}$ \\
\hline cohort [c2] & $\begin{array}{c}-0.088^{* * *} \\
(-0.133--0.044)\end{array}$ & $\begin{array}{c}-0.088^{* * *} \\
(-0.133--0.044)\end{array}$ & $\begin{array}{c}-0.088^{* * *} \\
(-0.133--0.044)\end{array}$ \\
\hline cohort [c3] & $\begin{array}{c}-0.085^{\text {*** }} \\
(-0.125--0.045)\end{array}$ & $\begin{array}{c}-0.087^{\text {*** }} \\
(-0.129--0.046)\end{array}$ & $\begin{array}{c}-0.087^{* * *} \\
(-0.129--0.046)\end{array}$ \\
\hline cohort [c4] & $\begin{array}{c}-0.144^{* * *} \\
(-0.186--0.103)\end{array}$ & $\begin{array}{c}-0.147^{* * *} \\
(-0.191--0.103)\end{array}$ & $\begin{array}{c}-0.147^{* * *} \\
(-0.191--0.103)\end{array}$ \\
\hline cohort [c5] & $\begin{array}{c}-0.181^{* * *} \\
(-0.234--0.127)\end{array}$ & $\begin{array}{c}-0.183^{* * *} \\
(-0.239--0.128)\end{array}$ & $\begin{array}{c}-0.183^{* * *} \\
(-0.239--0.128)\end{array}$ \\
\hline Test week & $\begin{array}{c}0.089^{* * *} \\
(0.087-0.091)\end{array}$ & $\begin{array}{c}0.089^{* * *} \\
(0.087-0.091)\end{array}$ & $\begin{array}{c}0.091^{* * *} \\
(0.089-0.093)\end{array}$ \\
\hline age [_s 7-8] & $\begin{array}{c}0.873^{* * *} \\
(0.845-0.900)\end{array}$ & $\begin{array}{c}0.873^{* * *} \\
(0.846-0.900)\end{array}$ & $\begin{array}{c}0.873^{* * *} \\
(0.846-0.900)\end{array}$ \\
\hline training_time [33] & $\begin{array}{c}0.199^{* * *} \\
(0.171-0.227)\end{array}$ & $\begin{array}{c}0.199^{* * *} \\
(0.170-0.227)\end{array}$ & $\begin{array}{c}0.199^{* * *} \\
(0.170-0.227)\end{array}$ \\
\hline $\begin{array}{l}\text { Test week * } \\
\text { training_time [33] }\end{array}$ & $\begin{array}{c}0.010^{* * *} \\
(0.008-0.012)\end{array}$ & $\begin{array}{c}0.010^{* * *} \\
(0.008-0.012)\end{array}$ & $\begin{array}{c}0.010^{* * *} \\
(0.008-0.012)\end{array}$ \\
\hline nvr_porportions & & $\begin{array}{c}0.109 \\
(-0.159-0.376)\end{array}$ & $\begin{array}{c}0.136 \\
(-0.132-0.404)\end{array}$ \\
\hline
\end{tabular}


rotcombi_porportions

Test week *nvr_amount

Test week *

rotcombi_amount

\section{Random Effects}

$\sigma^{2}$

$\tau_{00}$

$\tau_{11}$

$\rho_{01}$

ICC

$\mathrm{N}$

Observations

Marginal $\mathrm{R}^{2}$ /

Conditional $\mathrm{R}^{2}$

AIC

log-Likelihood
0.046

$$
(-0.102-0.194)
$$

$$
(-0.131-0.167)
$$

0.023 *

$(0.003-0.042)$

$-0.023^{* * *}$

$(-0.033--0.013)$
0.04

0.77 account_id

0.00 account_id.time

0.28 account_id

0.96

17648 account_id

52944

$0.225 / 0.969$

63540.653

$-31757.326$
$0.04 \quad 0.04$

0.77 account_id $\quad 0.77$ account_id

0.00 account_id.time $\quad 0.00$ account_id.time

0.28 account_id $\quad 0.28$ account_id

$0.96 \quad 0.96$

17648 account id $\quad 17648$ account id

52944

$\begin{array}{ll}0.224 / 0.969 & 0.225 / 0.969\end{array}$

$63543.801 \quad 63519.468$

$-31756.901 \quad-31742.734$ 


\section{Supplementary Table 5}

Linear model predicting interindividual improvement (i.e., slopes) while correcting for interindividual intercepts using training indices derived from the first week. P-values are FDR corrected. Level corresponds to the average correct level of the task within the first 5 days, while performance corresponds to the ratio of correct to total trials within the first 5 days.

\begin{tabular}{lcccc}
\hline \hline & \multicolumn{3}{c}{ Interindividual Improvement } \\
Predictors & std. Beta & standardized CI & $p$-FDR & vif \\
\hline Intercept) & -0.00 & $-0.01-0.01$ & 1 & - \\
Subj Math Factor Intercepts & 0.53 & $0.51-0.54$ & $2.2 \times 10^{-16}$ & 1.76 \\
Level NVR & 0.03 & $0.01-0.05$ & $1.7 \times 10^{-3}$ & 1.98 \\
Level Numberline & -0.12 & $-0.15--0.10$ & $9.3 \times 10^{-21}$ & 3.52 \\
Level Rotation & 0.00 & $-0.02-0.02$ & 1 & 2.03 \\
Level WM & -0.02 & $-0.04-0.01$ & 0.22 & 2.34 \\
Performance NVR & -0.01 & $-0.02-0.00$ & 0.22 & 1.18 \\
Performance Numberline & -0.02 & $-0.04-0.00$ & 0.17 & 2.02 \\
Performance Rotation & 0.02 & $0.00-0.04$ & 0.06 & 1.67 \\
Performance WM & 0.05 & $0.03-0.06$ & $9.7 \times 10^{-9}$ & 1.42 \\
\hline Observations & 16484 & & & \\
$\mathrm{R}^{2} / \mathrm{R}^{2}$ adjusted & $0.23 / 0.23$ & & & \\
\hline \hline
\end{tabular}

